\title{
ArcheoSciences
}

Revue d'archéométrie

41-1 | 2017

Varia

\section{Mise en évidence de cinq ateliers inédits d'amphores gauloises dans la région d'Arles}

Five new workshops of Gaulish amphorae in Arles' region

\section{Fabrice Bigot, Séverine Corbeel et Anne Schmitt}

\section{(2) OpenEdition}

12 Journals

Édition électronique

URL : https://journals.openedition.org/archeosciences/4902

DOI : 10.4000/archeosciences.4902

ISSN : 2104-3728

Éditeur

Presses universitaires de Rennes

\section{Édition imprimée}

Date de publication : 21 juin 2017

Pagination : 83-99

ISBN : 978-2-7535-5890-8

ISSN : 1960-1360

\section{Référence électronique}

Fabrice Bigot, Séverine Corbeel et Anne Schmitt, « Mise en évidence de cinq ateliers inédits d'amphores gauloises dans la région d'Arles », ArcheoSciences [En ligne], 41-1 | 2017, mis en ligne le 21 juin 2019, consulté le 27 janvier 2022. URL : http://journals.openedition.org/archeosciences/4902 ; DOI : https://doi.org/10.4000/archeosciences.4902 


\title{
Mise en évidence de cinq ateliers inédits d'amphores gauloises dans la région d'Arles ${ }^{*}$
}

\author{
Five New Workshops of Gaulish Amphorae in Arles' Region
}

\author{
Fabrice Bigot ${ }^{\mathrm{a}}$, Séverine CoRbeEL ${ }^{\mathrm{a}}$ et Anne SCHMitT ${ }^{\mathrm{b}}$ \\ avec la collaboration de Luc Long ${ }^{c}$ et David DJaOuI ${ }^{\mathrm{d}}$
}

\begin{abstract}
Résumé : Les recherches archéologiques et les analyses physico-chimiques conduites sur les amphores gauloises ces trente dernières années ont démontré le rôle important d'Arles et de la basse vallée du Rhône dans le commerce des amphores vinaires de Narbonnaise durant le Haut-Empire. De plus, plusieurs indices archéologiques (concentration d'amphores, terres cuites architecturales surcuites...) permettent de supposer l'existence d'un atelier péri-urbain dans cette agglomération portuaire, bien qu'aucun four n'ait encore été fouillé. Pour tester cette hypothèse, 76 amphores, issues des dépotoirs du Rhône et urbains, ont été analysées par fluorescence X. Puis, leur composition chimique a été comparée à celle des ateliers de Narbonnaise, étudiés au laboratoire de céramologie de Lyon (UMR5138, ARAR). Les résultats ont mis en évidence la possibilité de l'existence de cinq nouveaux ateliers localisés à Arles et/ou dans sa région. Ces derniers ont produits des amphores Gauloise 4 et Gauloise 5 entre la fin du $\mathrm{I}^{\text {er }}$ siècle apr. J.-C. et le $\mathrm{III}^{\mathrm{e}}$ siècle apr. J.-C.
\end{abstract}

\begin{abstract}
For thirty years, archaeological and chemical analyses on Gauloise amphorae have demonstraded the important place of Arles and the lower Rhône valley in the exchange of wines amphorae from the province of Narbonensis during the Early Roman Empire. Moreover, many archaeological indices (concentration of amphorae, architectural terra-cotta...) have suggested the presence of a suburban workshop in Arles, even if no kiln has been excavated. In order to test this hypothesis, 76 amphorae from harbor's dumping grounds in the Rhône aswellas urban deposits were analyzed by X-ray fluorescence. Then, their chemical composition was compared to the Narbonensis workshops, which were already studied at the "laboratoire de céramologie de Lyon" (UMR5138, ARAR). The results pointed out the possibility of five new workshops in Arles andlor nearby. These ones produced Gauloise 4 and Gauloise 5 amphorae between the end of the $1^{\text {st }}$ century A.C and the $3^{\text {rd }}$ century A.C.
\end{abstract}

Mots clés : Amphores gauloises, Arles, Céramologie, Commerce antique, Groupes de référence géochimiques.

Keywords: Gauloise amphorae, Arles, Ceramology, Antic trade, Geochemical reference groups.

\footnotetext{
* Ce travail est inscrit dans l'axe thématique "Économie et environnement » du programme scientifique du Labex ARCHIMEDE, programme IA-ANR-11-LABX-0032-01.

a Doctorant, ASM-Archéologie des Sociétés Méditerranéennes, UMR 5140, université Paul-Valéry Montpellier, CNRS, MCC, 34000 MONTPELLIER, France.(fabrice-bigot@hotmail.fr) (severine_1989@hotmail.com)

b Directrice de recherche, ARAR-Archéologie et Archéométrie, UMR5138, MSH MOM, université Lumière - Lyon 2, CNRS, 69000 LYON, France. (Anne.schmitt@mom.fr)

' Conservateur en chef du patrimoine, ASM-Archéologie des Sociétés Méditerranéennes, UMR 5140, université Paul-Valéry Montpellier, CNRS, MCC, 34000 MONTPELLIER,France. (Luc.long@wanadoo.fr)

'Archéologue territorial au musée départemental de l'Arles antique, Conseil départemental des Bouches-du-Rhône.
} 


\section{INTRODUCTION}

La viticulture spéculative occupe une place très importante dans le développement économique de la Narbonnaise entre la fin du I $^{\text {er }}$ siècle av. J.-C. et le Iv $^{\mathrm{e}}$ siècle apr. J.-C. (Brun, Laubenheimer, 2001 : 206-208). Au Haut-Empire, cette région représente la première province exportatrice de vin en amphore, consommé sur le limes germanique et en Bretagne (Baudoux, 1996 : 62; Fitzpatrick, 1992 : 181). Elle constitue également l'une des principales pourvoyeuses en vin de Rome et d'Ostie (Panella, 1992; Panella, Rizzo, 2014 : 81). Ces observations ont été permises grâce aux recherches conduites sur les amphores gauloises, initiées par F. Laubenheimer en 1985 (Laubenheimer, 1985). La multiplication des études, depuis une trentaine d'années, a permis d'étoffer nos connaissances sur la géographie des ateliers et les courants commerciaux de ces conteneurs. Néanmoins, les dernières synthèses mettent en exergue un déficit d'ateliers et les difficultés de déterminer la région de provenance de ces amphores de Narbonnaise, à partir d'une simple analyse visuelle de leur pâte (Mauné, 2013; Laubenheimer, Schmitt, 2009). Elles montrent cependant l'apport considérable que constituent les analyses physico-chimiques pour la mise en évidence de l'origine des amphores et des ateliers (Mauné, 2013 : 357 ; Laubenheimer, Schmitt, 2009). L'existence d'une zone de production majeure dans la basse vallée du Rhône a ainsi été démontrée, bien que les ateliers restent profondément méconnus dans ce secteur (Ibid: 154).

Les contextes portuaires et de consommation d'Arles sont en revanche bien documentés, en particulier grâce aux fouilles subaquatiques menées dans le Rhône par Luc Long (DRASSM) depuis une trentaine d'années et par les équipes du musée de l'Arles Antique (Conseil général des Bouchesdu-Rhône) depuis 2008. Ces opérations ont permis la mise au jour d'un imposant dépotoir portuaire dans lequel les amphores gauloises vinaires sont prépondérantes entre la période flavienne et le début du Iv ${ }^{\mathrm{e}}$ s. apr. J.-C. (Duperron, 2014 : 99-100) ${ }^{1}$ (figure 1). À titre d'exemple, les fouilles du dépotoir recouvrant le chaland Arles-Rhône 3 ont livré 1679 amphores gauloises soit $60 \%$ des amphores et la fouille de sauvetage dite " de la CNR » 202 conteneurs gaulois soit $77,5 \%$ des amphores et $90 \%$ des conteneurs vinaires (Bigot, Djaoui, 2013 : 377; Duperron, 2014, vol. 1 : 97; vol. 2 : 195-196). À proximité immédiate, le site ArlesRhône 1 est presque exclusivement composé d'amphores Gauloise 4: " au total plus d'une centaine de cols et une

1. Le mobilier de ces dépotoirs a été étudié par Guillaume Duperron dans le cadre d'une thèse sous la direction de S. Mauné (UMR 5140) et M. Poux (UMR5138), soutenue à l'université Paul-Valéry/Montpellier 3. (Duperron, 2014). dizaine d'amphores entières ont été répertoriées ", dont six portent la marque T.CR.V'IT'. L. Long suppose qu'elle pourrait correspondre à un atelier d'Arles ou de sa région (Long, 1992 : 11). De plus, "il est à noter la présence de briques et d'importants rebuts de cuisson provenant d'un four de tuilier" (ibid. : 11). Les concentrations d'amphores sur ces trois sites nous conduisent plutôt à proposer la présence d'un ou plusieurs ateliers amphoriques périurbains. Cette hypothèse est étayée par le grand nombre d'estampilles d'une même marque découvertes dans ces dépotoirs ${ }^{2}$, des estampilles pour lesquelles l'atelier de fabrication reste inconnu, bien que l'origine rhodanienne de certaines soit avérée ${ }^{3}$ (Laubenheimer, Schmitt, 2009 : 129-130) et des estampilles inédites M.A.I et MFM (Duperron, 2014 : 147; Bigot, Djaoui, 2013 : 389; Corbeel, Duperron, 2013 : 422). Toutes ces estampilles permettent d'émettre l'hypothèse de l'existence d'un important centre de production composé de plusieurs officines ${ }^{4}$ (ibid.).

Ces ateliers auraient produit majoritairement des Gauloise 4, destinées au grand commerce (Laubenheimer, 2001 : 55). Létude de ces amphores et la détermination de leur origine sont donc capitales pour mieux appréhender l'impact de la basse vallée du Rhône dans les échanges vers les centres de consommation de Méditerranée 5 . De plus, les timbres mis au jour à Arles, dont l'origine locale est envisagée, constituent un corpus exceptionnel pour comprendre l'organisation de la production d'amphores et du conditionnement

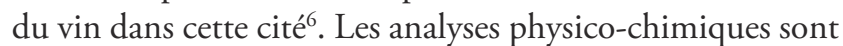
essentielles pour vérifier l'hypothèse de l'existence d'un centre de production à Arles.

2. On dénombre 18 exemplaires T.CR.V'IT', dont neuf appartenant au gisement Arles-Rhône 1/CNR, et 12 exemplaires A.P.M, dont neuf appartenant au gisement $\mathrm{A} /$ Arles-Rhône 3,

3. On comptabilise six exemplaires MATVR/MATVRI, quatre exemplaires ALBIN, deux exemplaires C.D.V; deux exemplaires C.M.S, et une occurrence des marques S.A.D, Q.C.H, IVLI./ZOSIMI, M.I.P, S.P.S, L.SE, T.V.P et MERCATOR.

4. Les ateliers de Dr. 20 de Bétique fournissent ce type de cas de figure. À titre d'exemple, dans l'atelier de Las Delicias à Ecija (Province de Séville), par la première phase datée du $\mathrm{I}^{\mathrm{er}}$ s. apr. J.-C., environ cinq figlinae semblent être en activité (Mauné et al., 2014, p. 423).

5. Ce travail fait l'objet d'une thèse préparée par F. Bigot, dirigée par S. Mauné (DR CNRS, UMR 5140) et F. Laubenheimer (DR émérite CNRS, UMR 7041) et intitulée " Nouvelles données, nouvelles réflexions sur la production et la diffusion des amphores gauloises à partir des contextes portuaires et littoraux de Gaule Narbonnaise ( ${ }^{\text {er }}$ s. av. J.-C.IV ${ }^{e}$ s. apr. J.-C.) ».

6. Ce travail fait l'objet d'une thèse préparée par $S$. Corbeel, dirigée par S. Mauné (DR CNRS, UMR 5140) et M.-B. Carre (CR CNRS, UMR 7299) et intitulée "Les producteurs de tuiles et d'amphores de Gaule Narbonnaise. L'apport des estampilles à la connaissance des structures économiques d'une province romaine ( $\mathrm{I}^{\mathrm{er}} \mathrm{s}$. av. J.-C.-début $\mathrm{IV}^{\mathrm{e}}$ s. apr. J.-C. ». 
Figure 1 : Carte de localisation des sites étudiés à Arles (IGN 2016 - [www.geoportail.gouv.fr]); $\mathrm{n}^{\circ} 1$ : Arles-Rhône 1/ CNR; n 2 : Gisement A/Arles-Rhône 3; $\mathrm{n}^{\circ} 3$ Brossolette; $\mathrm{n}^{\circ} 4$ : La Verrerie; $\mathrm{n}^{\circ} 5$ : Cimetière de Trinquetaille; $\mathrm{n}^{\circ} 6$ La Pointe; $\mathrm{n}^{\circ} 7$ : Place Jean-Baptise Massillon; $\mathrm{n}^{\circ} 8$ : Cirque.

Figure 1: Map of the sites in Arles (IGN 2016 - www.geoportail.gouv.fr); $n^{\circ} 1$ : Arles-Rhône 1/CNR; $n^{\circ} 2$ : Gisement A/ Arles-Rhône 3; $n^{\circ} 3$ Brossolette; $n^{\circ}$ 4: La Verrerie; $n^{\circ}$ 5: Cimetière de Trinquetaille; $n^{\circ}$ 6: La Pointe; $n^{\circ}$ 7: Place Jean-BaptiseMassillon; $n^{\circ}$ 8: Cirque.

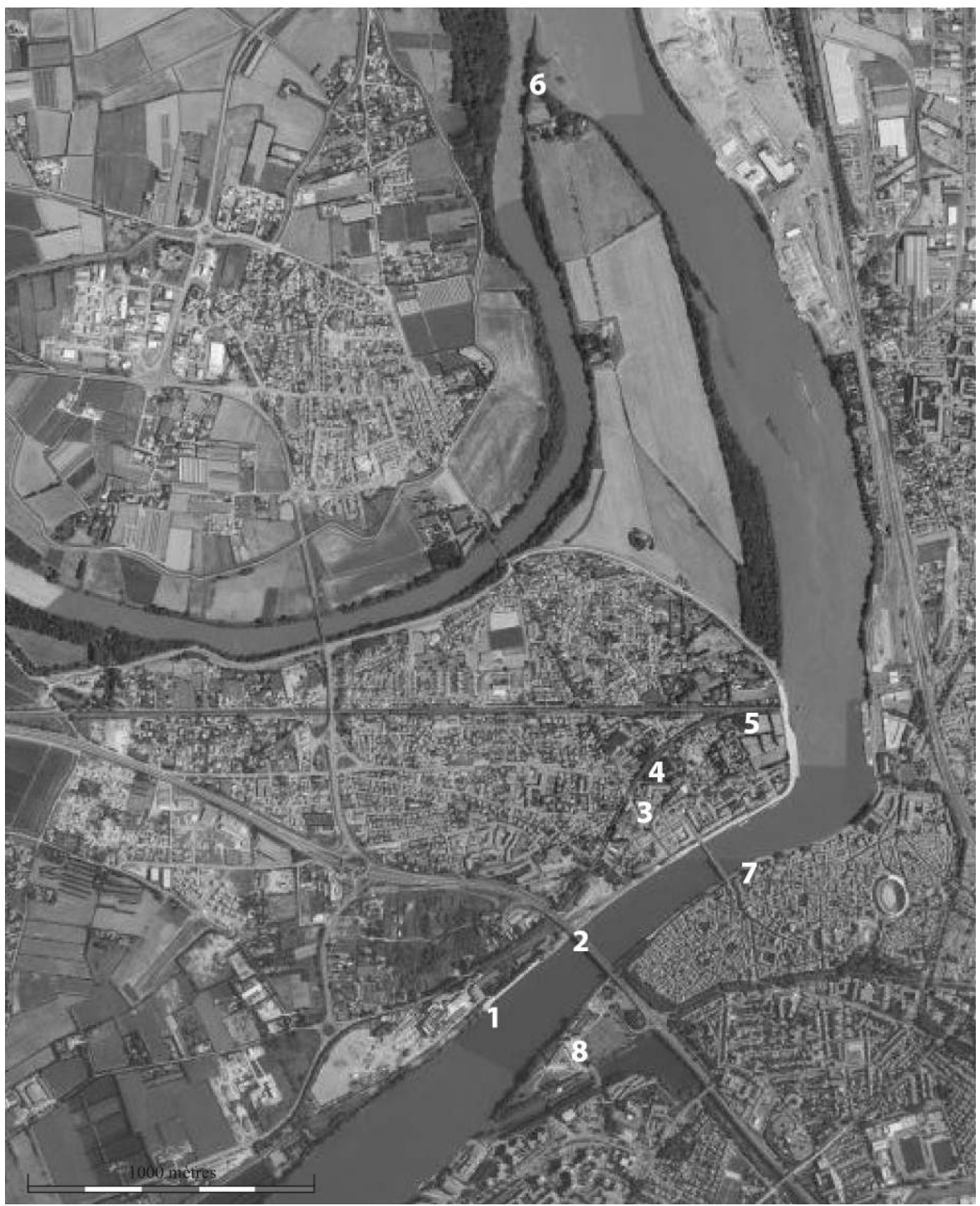

\section{LES ANALYSES PHYSICO-CHIMIQUES}

Les analyses chimiques ont été réalisées par fluorescence $\mathrm{X}$ en dispersion de longueur d'onde au laboratoire de céramologie de l'UMR 5138 ARAR de Lyon. La comparaison des compositions chimiques a été effectuée par classification ascendante hiérarchique à partir des distances euclidiennes non pondérées sur variables centrées réduites relatives aux 16 constituants chimiques suivants: $\mathrm{CaO}, \mathrm{Fe}_{2} \mathrm{O}_{3}, \mathrm{TiO}_{2}$, $\mathrm{K}_{2} \mathrm{O}, \mathrm{SiO}_{2}, \mathrm{Al}_{2} \mathrm{O}_{3}, \mathrm{MgO}, \mathrm{MnO}, \mathrm{Zr}, \mathrm{Sr}, \mathrm{Zn}, \mathrm{Rb}, \mathrm{Cr}, \mathrm{Ni}$, $\mathrm{V}$, Ce. Le baryum $(\mathrm{Ba})$, le sodium $\left(\mathrm{Na}_{2} \mathrm{O}\right)$ et le phosphore $\left(\mathrm{P}_{2} \mathrm{O}_{5}\right)$ ne sont pas utilisés dans les calculs pour s'affranchir de l'influence d'une éventuelle pollution-contamination lors de l'enfouissement (Picon, 1976). Le graphique obtenu représente le degré de ressemblance entre les différents exem- plaires et permet de visualiser les groupes de composition. Il faut ensuite vérifier la validité des classifications obtenues en s'attachant à repérer à tous les stades les individus qui pourraient être étrangers. Il faut tenir compte des dispersions de composition au sein des ateliers, de l'interprétation géochimique des variations et bien sûr des altérations de compositions chimiques qui ont pu intervenir lors de l'enfouissement (Buxeda i Garrigós et al., 2002; Schwedt et al., 2006).

La conservation en milieu humide pouvant altérer la composition des pâtes des amphores, nous avons d'abord vérifié si les amphores découvertes dans le Rhône avaient subi ou non des altérations de leur composition chimique. L'échantillonnage a porté sur dix Gauloise 4 du dépotoir Arles-Rhône 3 (dont six timbrées A.P.M, une T.CR.V'IT', 
une C.M.S et une [M.I.]P) et dix Gauloise 4 issues de contextes terrestres (huit amphores appartenant à un dépotoir de berge place Jean Baptiste Massillon dont une timbrée MARTIAL; une amphore du site urbain de la Verrerie à Trinquetaille; une amphore timbrée C.D.V du site urbain Pierre-Brossolette ${ }^{7}$ ). Aucune différence notable dans la composition n'a été observée, c'est pourquoi on peut considérer que le séjour des amphores dans l'eau du Rhône ne génère pas d'altération des compositions des pâtes.

\section{Définition du groupe arlésien}

\section{Les amphores analysées}

Les amphores Gauloise 4 de Narbonnaise, produites à partir de la période flavienne, ont en commun une pâte beige claire à orangée, poreuse et fréquemment friable. Les inclusions visibles sont rares et le plus souvent quartzeuses. En général, il est très difficile de faire à l'œil nu une distinction entre les ateliers d'après la pâte, il en est de même à la loupe car les inclusions sont de petite taille et peu caractéristiques (Laubenheimer, Schmittn 2009 : 39). Il est donc nécessaire d'avoir recourt à des analyses physico-chimiques pour confirmer ou infirmer les hypothèses posées par l'étude céramologique.

Le corpus comprend les 20 Gauloise 4 utilisées pour le test de pollution, décrites ci-dessus, ainsi que 33 amphores du gisement $\mathrm{A} /$ Arles-Rhône 3, dont sept timbrées (quatre exemplaires A.P.M; MFM; T.V.P; S.P.S) (figure 2), 11 Gauloise 4 originaires des gisements Arles-Rhône 1 et CNR estampillées (neuf exemplaires T.CR.V'IT'; MATVRI; A.P.M) (figure 3) et deux Gauloise 4 timbrées A.P.M découvertes en prospection dans la zone 420 du Rhône et lors des fouilles du Cirque en 1987. Nous avons également inclus à notre étude deux amphores timbrées C.M.S et MATVRI, découvertes lors des fouilles du Cirque d'Arles en 1974 (Laubenheimer, Schmitt, 2009 : 151). Le corpus des amphores analysées pour vérifier l'existence d'un atelier à Arles se compose donc de 68 Gauloise $4^{8}$. Ces conteneurs sont datés entre la période flavienne et le milieu du $\mathrm{II}^{\mathrm{e}}$ siècle apr. J.-C., hormis sept amphores provenant de la place Jean-Baptiste Massillon dont la chronologie est comprise entre le milieu du $\mathrm{II}^{\mathrm{e}}$ siècle et le $\mathrm{III}^{\mathrm{e}}$ siècle apr. J.-C., ainsi que quelques amphores mar-

7. Nous remercions A. Charon (conservateur en chef du musée de l'Arles Antique), M.-P. Rothé (Archéologue territorial au musée départemental de l'Arles antique, CG Bouches-du Rhône) responsable de la fouille de la Verrerie et D. Isoardi (CNRS, UMR 7299), responsable de la fouille de la place Jean-Baptiste Massillon pour nous avoir permis l'étude de ces céramiques.

8. Ces analyses ont été réalisées grâce au financement du Labex Archimède, programme IA-ANR-11-LABX-0032-01.

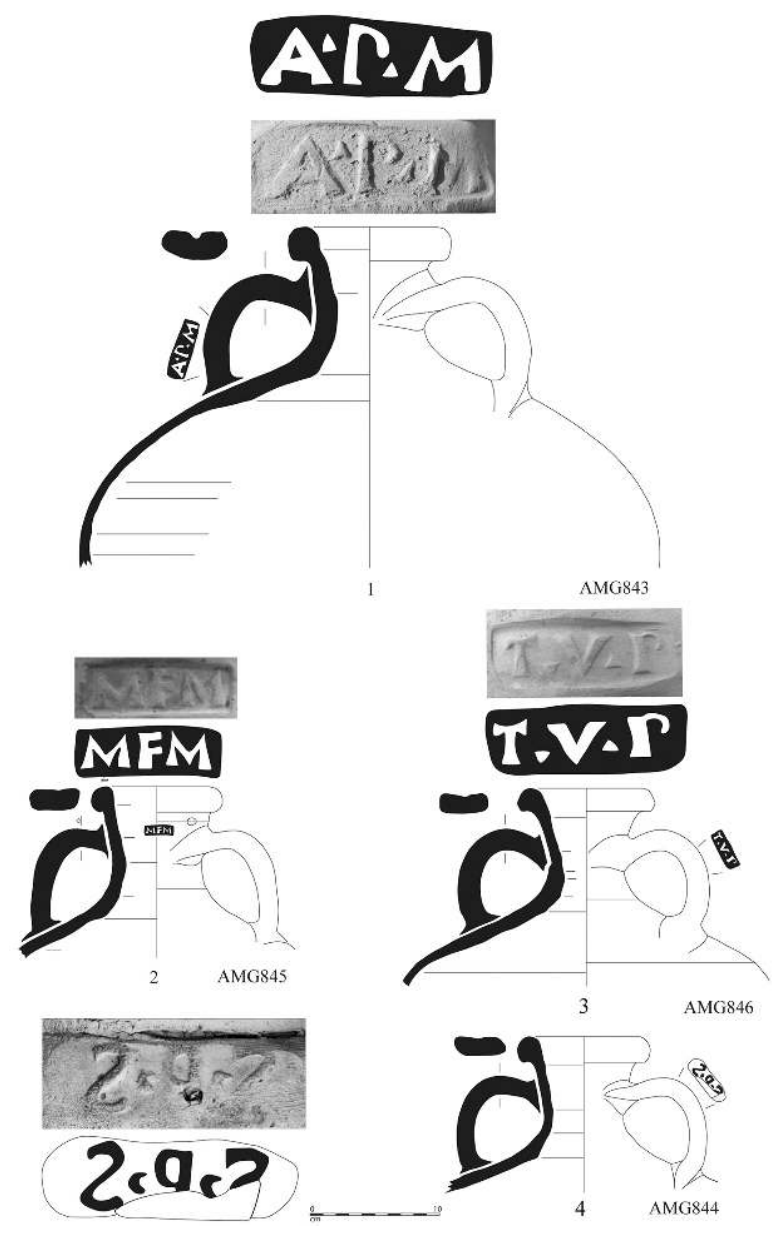

Figure 2 : (Voir planche couleur X) Amphores Gauloise 4 estampillées analysées.

Figure 2: (See colour plate X) Analysed Gauloise 4 Amphorae with marks.

quées du Cirque et des gisements Arles-Rhône 1 et CNR, datées de la seconde moitié du II $^{\mathrm{e}}$ siècle.

Les exemplaires ont été sélectionnés suite à une observation minutieuse de leur pâte à l'œil nu. Cette dernière est fine, calcaire, dure ou savonneuse, de couleur beige à orangée et contient des particules blanches, noires et du mica dont les quantités et les dimensions varient de façon non uniforme de très faibles à moyennes. Deux groupes se distinguent par la présence ou l'absence de petites inclusions rouges correspondant probablement à des oxydes de fer. Le groupe le plus important (groupe A) contient des inclusions d'oxydes. Il est formé de 51 individus ${ }^{9}$ contre 13 exemplaires

9. Les estampilles A.P.M (10 individus), C.M.S, S.P.S, MFM, T.V.P, M.I.P, T.CR.V'IT' (cinq individus) et MATVRI présentent une pâte de ce type. 


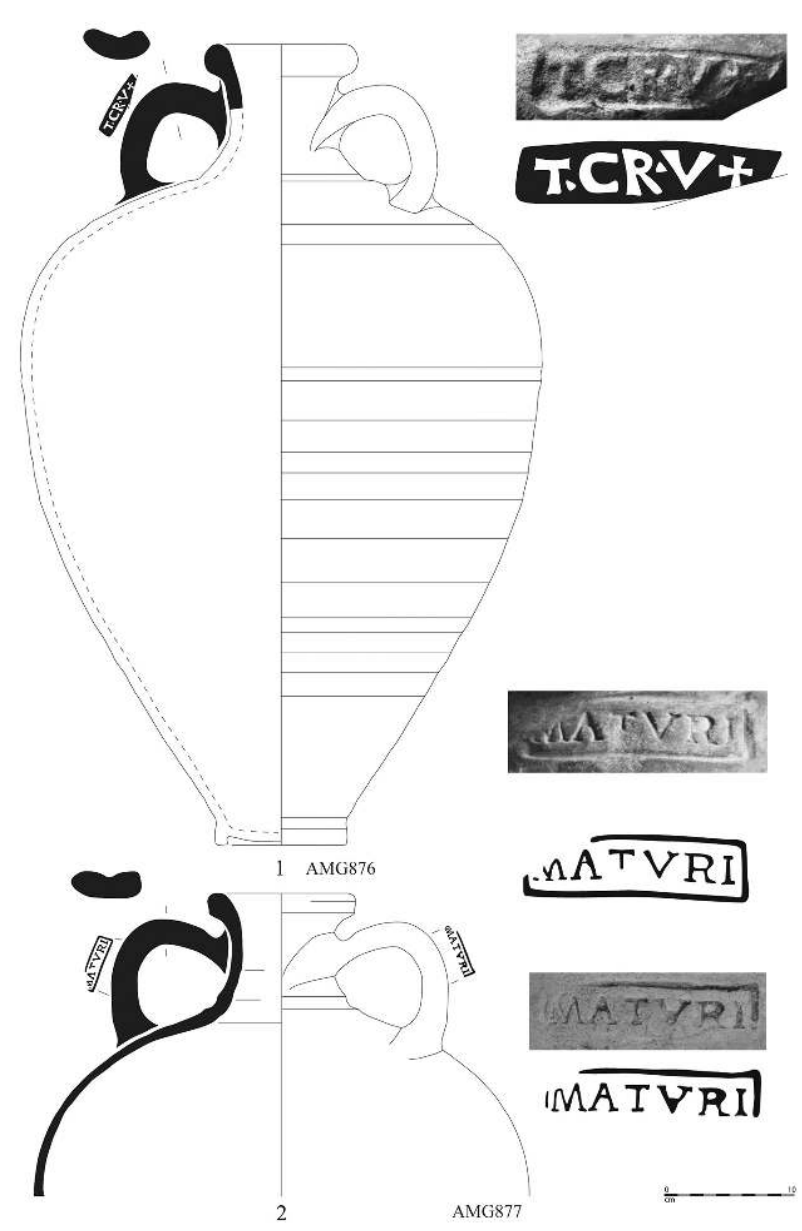

Figure 3 : (Voir planche couleur XI) Amphores Gauloise 4 estampillées analysées.

Figure 3: (See colour plate XI) Analysed Gauloise 4 Amphorae with marks.

seulement dans le second groupe ${ }^{10}$ (groupe B). Qui plus est, on recense un exemplaire atypique (AMG830) dont la pâte est de couleur rouge/rosée. Elle contient des inclusions blanches, noires, plus rarement rouges et un très grand nombre de particules de mica. Cet individu a été incorporé car il porte une marque A.P.M, que nous supposions locale (Bigot, Djaoui, 2013 : 389; Corbeel, Duperron, 2013 : p. 422). Du fait de la nature très fine de la pâte, nous n'avons pas effectué d'analyse plus poussée au microscope pétrographique.

\section{Résultats des analyses}

Les amphores de type Gauloise 4 découvertes à Arles présentent une pâte argileuse dont les taux de calcaire varient

10. Cinq exemplaires timbrés T.CR.V'IT', un exemplaire A.P.M et le timbre $[\mathrm{MI}] \mathrm{P}$ possèdent une pâte de ce type. en moyenne de 10 à $16 \%$. La classification d'après les données chimiques montre une répartition en cinq groupes et la présence de nombreux marginaux (figure 4). Il est possible qu'un seul vaste centre de production ait utilisé différentes argiles. Cette situation est attestée dans l'officine de Saint-Bézard à Aspiran, où deux types de pâte sont recensés (Laubenheimer, Schmitt, 2009 : 51-52, 58). Néanmoins, il nous semble plus probable de restituer autant d'ateliers que de groupes, bien que les différences de compositions chimiques soient minimes (tableau 2). On peut émettre l'hypothèse que la matière première argileuse utilisée par les potiers provient de la même formation géologique, les différences entre les ateliers pouvant s'expliquer par des niveaux ou des lieux de prélèvements différents ainsi que des modes de préparation de la pâte céramique propre à chaque atelier.

En dehors de ces groupes, deux exemplaires se placent en position de marginal interne (AMG818 et AMG847) avec une composition intermédiaire entre les groupes 4 et 5 (figure 4). Enfin, neuf individus se placent en position totalement marginale. Deux d'entre-eux (AMG 825 et AMG 840) ont des taux de $\mathrm{CaO}$ bien supérieurs à tous les autres exemplaires, il s'agit vraisemblablement d'importations. Pour les autres exemplaires, il est parfois difficile d'identifier les raisons de leur classement marginal (AMG849, AMG815, AMG820, AMG886, AMG874, AMG839, AMG821), les principales variations portent sur les taux de $\mathrm{CaO}, \mathrm{Fe}_{2} \mathrm{O}_{3}$ et $\mathrm{MnO}$. En l'absence d'analyse de prélèvement d'argile, il est difficile de dire si ces différences peuvent s'expliquer par des variations au sein des gisements d'argiles locales. Ces individus ne formant pas un groupe cohérent, il est plus probable qu'il s'agisse d'importations isolées.

Il convient à présent de mettre en relation ces résultats avec les données archéologiques. En premier lieu, signalons que les exemplaires initialement répartis en deux ensembles A et $B$, d'après les aspects macroscopiques des pâtes, se dispersent ici parmi cinq groupes de compositions chimiques et les 10 individus marginaux. L'amphore Gauloise 4 à pâte macroscopiquement atypique (AMG830) se place au sein du groupe 2 .

Le groupe 2 est largement majoritaire avec 32 exemplaires, parmi lesquels sept marques T.CR.V'IT', quatre estampilles A.P.M ainsi que les timbres T.V.P, C.M.S, C.D.V, MFM et MATVRI. L'effectif important de ce groupe suggère une origine arlésienne des amphores qui y sont classées. Les quatre autres groupes, plus modestes, comprennent certaines amphores estampillées; le timbre A.P.M est recensé dans les groupes 1, 2, 3 et 4 et sa matrice A est présente dans chacun d'entre eux. Les marques MATVRI et MART[IAL] sont attestées dans le groupe 5, l'estampille T.CR.V'IT' dans les groupes 2, 3 et 4, les timbres S.P.S et CMS dans le groupe 3 


\begin{tabular}{|c|c|c|c|c|}
\hline Numéro d'analyse & Site & Numéro d'Inventaire & Timbre & Matrice \\
\hline AMG598 & Cirque & CIR.74.00.1007 & MATVRI & $\mathrm{D}$ \\
\hline AMG601 & Cirque & CIR.74.00.2470 & C.M.S & $\mathrm{A}$ \\
\hline AMG806 & Arles-Rhône 3 & AR3-2004-36 & A.P.M & $\mathrm{A}$ \\
\hline AMG807 & Arles-Rhône 3 & AR3-3001-747 & C.M.S & $\mathrm{B}$ \\
\hline AMG808 & Arles-Rhône 3 & AR3-2004-176 & A.P.M & $\mathrm{D}$ \\
\hline AMG809 & Arles-Rhône 3 & AR3-2001-206 & [M.I.]P & A ou B \\
\hline AMG810 & Arles-Rhône 3 & AR3-2031-17 & - & - \\
\hline AMG811 & Arles-Rhône 3 & AR3-2004-177 & - & - \\
\hline AMG812 & Arles-Rhône 3 & AR3-3020-257 & T.CR.V'IT' & $\mathrm{A}$ \\
\hline AMG813 & Arles-Rhône 3 & AR3-2004-37 & A.P.M & $\mathrm{A}$ \\
\hline AMG814 & Arles-Rhône 3 & AR3-4001-290 & - & \\
\hline AMG815 & Arles-Rhône 3 & AR3-2001-387 & A.P.M & $\mathrm{C}$ \\
\hline AMG816 & Jean-Baptiste Massillon & JBM09-1072 & - & - \\
\hline AMG817 & Jean-Baptiste Massillon & JBM09-1064-3 & - & - \\
\hline AMG818 & Jean-Baptiste Massillon & JBM09-1068-1 & - & - \\
\hline AMG819 & Jean-Baptiste Massillon & JBM09-1064-2 & - & - \\
\hline AMG820 & Jean-Baptiste Massillon & JBM09-1087 & - & - \\
\hline AMG821 & Jean-Baptiste Massillon & JBM09-1091-10 & - & - \\
\hline AMG822 & Jean-Baptiste Massillon & JBM09-1068 & - & - \\
\hline AMG823 & Jean-Baptiste Massillon & JBM09-1023-14 & MARTIAL & - \\
\hline AMG824 & Brossolette & BRO-G4-1 & C.D.V & $\mathrm{D}$ \\
\hline AMG825 & La Verrerie & VER13.9 & - & - \\
\hline AMG826 & Arles-Rhône 3 & AR3-2029-236 & - & - \\
\hline AMG827 & Arles-Rhône 3 & AR3-2018-10 & - & - \\
\hline AMG828 & Arles-Rhône 3 & AR3-3007-150 & - & - \\
\hline AMG829 & Arles-Rhône 3 & AR3-3001-622 & - & - \\
\hline AMG830 & Arles-Rhône 3 & AR3-C2-366 & A.P.M & $\mathrm{A}$ \\
\hline AMG839 & Zone 420 & RH.09.Z420.S3.293 & A.P.M & $\mathrm{A}$ \\
\hline AMG840 & Gisement A & RH.04.00.05.1077 & A.P.M & $\mathrm{A}$ \\
\hline AMG841 & Arles-Rhône 1 & RH.89/90-AR1-8 & A.P.M & $\mathrm{A}$ \\
\hline AMG842 & Arles-Rhône 3 & AR3-C2-64 & A.P.M & $\mathrm{A}$ \\
\hline AMG843 & Arles-Rhône 3 & AR3-2007-113 & A.P.M & $\mathrm{A}$ \\
\hline AMG844 & Arles-Rhône 3 & AR3-2007-285 & S.P.S & $\mathrm{B}$ \\
\hline AMG845 & Arles-Rhône 3 & AR3-3001-727 & MFM & \\
\hline AMG846 & Arles-Rhône 3 & AR3-2003-43 & T.V.P & $\mathrm{A}$ \\
\hline AMG847 & Arles-Rhône 3 & AR3-2029-28 & - & - \\
\hline AMG848 & Arles-Rhône 3 & AR3-2031-33 & - & - \\
\hline AMG849 & Arles-Rhône 3 & AR3-3007-224 & - & - \\
\hline AMG850 & Arles-Rhône 3 & AR3-3007-225 & - & - \\
\hline AMG851 & Arles-Rhône 3 & AR3-1007-6 & - & - \\
\hline AMG852 & Arles-Rhône 3 & AR3-1007-9 & - & - \\
\hline AMG853 & Arles-Rhône 3 & AR3-2005-41 & - & - \\
\hline AMG854 & Arles-Rhône 3 & AR3-2018-16 & - & - \\
\hline AMG855 & Arles-Rhône 3 & AR3-3020-252 & - & - \\
\hline AMG856 & Arles-Rhône 3 & AR3-C2-24 & - & - \\
\hline AMG857 & Arles-Rhône 3 & AR3-2003-52 & - & - \\
\hline AMG858 & Arles-Rhône 3 & AR3-C1-64 & - & - \\
\hline AMG859 & Arles-Rhône 3 & AR3-3007-182 & - & - \\
\hline AMG860 & Arles-Rhône 3 & AR3-3007-185 & - & - \\
\hline AMG861 & Arles-Rhône 3 & AR3-2029-97 & - & - \\
\hline AMG862 & Arles-Rhône 3 & AR3-2029-98 & - & - \\
\hline AMG864 & Arles-Rhône 3 & AR3-2029-94 & - & - \\
\hline AMG865 & Arles-Rhône 3 & AR3-3007-182 & - & - \\
\hline AMG866 & Arles-Rhône 3 & AR3-3007-186 & - & - \\
\hline AMG867 & Arles-Rhône 3 & AR3-3007-176 & - & - \\
\hline AMG868 & Arles-Rhône 3 & AR3-2029-244 & - & - \\
\hline AMG869 & Arles-Rhône 3 & AR3-3007-228 & - & - \\
\hline AMG870 & Arles-Rhône 1 & RH.89/90-AR1-1 & T.CR.V'IT' & A ou $\mathrm{C}$ \\
\hline AMG871 & Arles-Rhône 1 & RH.89/90-AR1-2 & T.CR.V'IT' & A ou $\mathrm{C}$ \\
\hline AMG872 & Arles-Rhône 1 & RH.89/90-AR1-3 & T.CR.V'IT' & A ou C \\
\hline AMG873 & Arles-Rhône 1 & RH.89/90-AR1-4 & T.CR.V'IT' & A ou C \\
\hline AMG874 & Arles-Rhône 1 & RH.89/90-AR1-5 & T.CR.V'IT' & A ou C \\
\hline AMG875 & Arles-Rhône 1 & RH.89/90-AR1-6 & T.CR.V'IT' & A ou C \\
\hline AMG876 & CNR & RH.07.CNR.PVI.466 & T.CR.V'IT' & A ou C \\
\hline AMG877 & Arles-Rhône 1 & RH.89/90-AR1-7 & MATVRI & $\mathrm{A}$ \\
\hline AMG880 & CNR & RH.07.CNR.PIII.467 & T.CR.V'IT' & A \\
\hline AMG881 & CNR & RH.07.CNR.PVI.469 & T.CR.V'IT' & A ou C \\
\hline AMG886 & Cirque, fouille de 1987 & CIR.87.255 & A.P.M & A, B ou D \\
\hline
\end{tabular}

Tableau $1:$ Liste des amphores Gauloise 4 analysées par fluorescence $\mathrm{X}$.

Table 1: List of amphorae Gauloise 4 analyzed by XRF. 


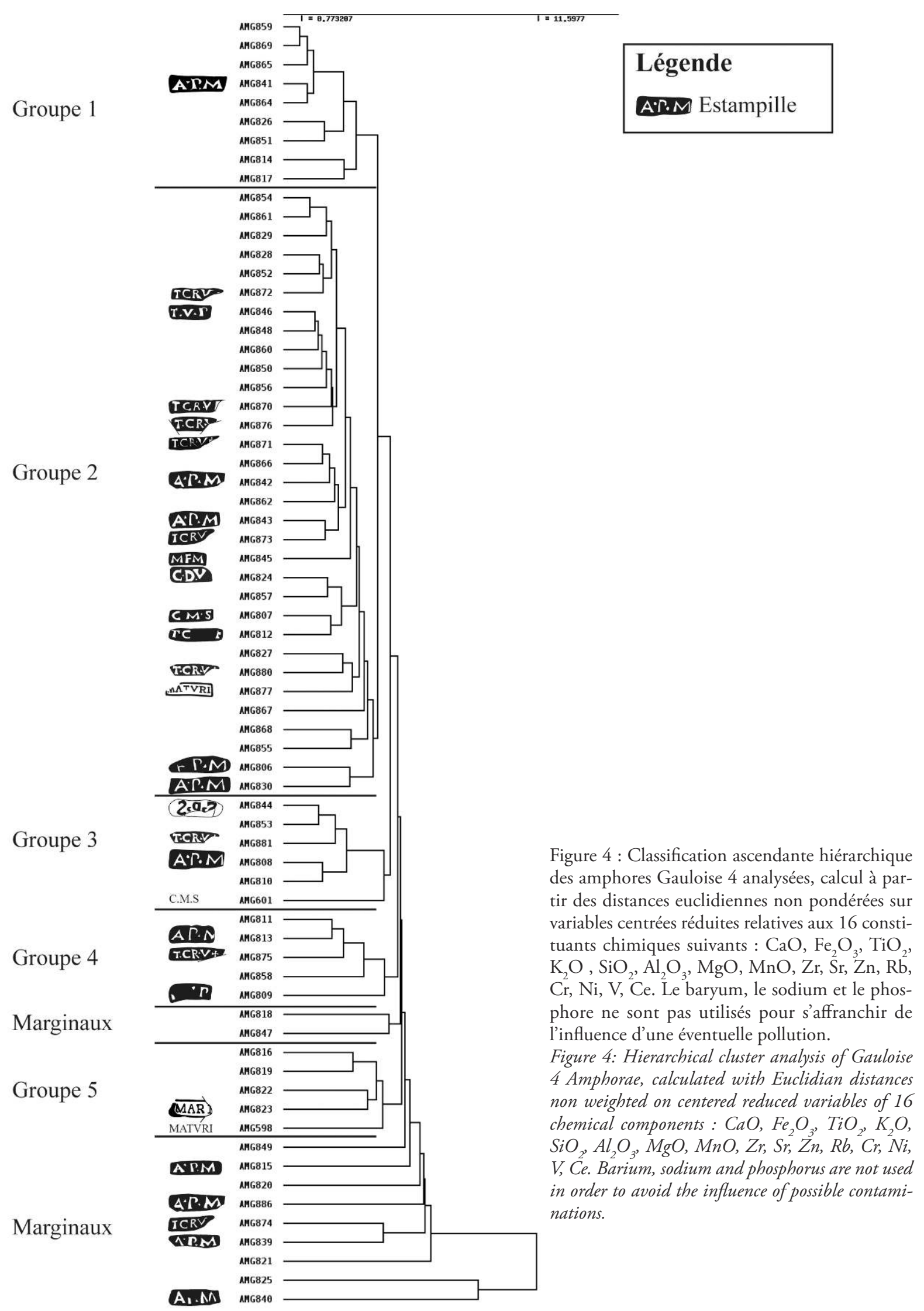




\begin{tabular}{|c|c|c|c|c|c|c|c|c|c|c|c|c|c|c|c|c|c|}
\hline & & $\mathrm{CaO}$ & $\mathrm{Fe}_{2} \mathrm{O}_{3}$ & $\mathrm{TiO}_{2}$ & $\mathrm{~K}_{2} \mathrm{O}$ & $\mathrm{SiO}_{2}$ & $\mathrm{Al}_{2} \mathrm{O}_{3}$ & $\mathrm{MgO}$ & $\mathrm{MnO}$ & $\mathrm{Zr}$ & $\mathrm{Sr}$ & $\mathrm{Rb}$ & $\mathrm{Zn}$ & $\mathrm{Cr}$ & $\mathrm{Ni}$ & V & $\mathrm{Ce}$ \\
\hline \multirow{3}{*}{$\begin{array}{l}\text { Groupe } 1 \\
\mathrm{n}=9\end{array}$} & $\mathrm{~m}$ & 13,65 & 6,33 & 0,698 & 3,32 & 55,46 & 16,74 & 2,59 & 0,1052 & 130 & 484 & 170 & 107 & 111 & 68 & 112 & 74 \\
\hline & $\sigma$ & 1,29 & 0,41 & 0,009 & 0,20 & 1,59 & 0,46 & 0,22 & 0,0097 & 9 & 27 & 9 & 4 & 3 & 4 & 5 & 7 \\
\hline & $\sigma \%$ & 9 & 6 & 1 & 6 & 3 & 3 & 8 & 9 & 7 & 6 & 5 & 4 & 3 & 6 & 4 & 9 \\
\hline \multirow{3}{*}{$\begin{array}{l}\text { Groupe } 2 \\
\mathrm{n}=32\end{array}$} & $\mathrm{~m}$ & 15,25 & 6,11 & 0,688 & 2,74 & 56,41 & 15,38 & 2,38 & 0,1230 & 151 & 473 & 139 & 99 & 103 & 64 & 110 & 73 \\
\hline & $\sigma$ & 1,94 & 0,53 & 0,020 & 0,23 & 1,54 & 0,36 & 0,26 & 0,0191 & 13 & 34 & 13 & 4 & 4 & 4 & 10 & 6 \\
\hline & $\sigma \%$ & 13 & 9 & 3 & 8 & 3 & 2 & 11 & 16 & 9 & 7 & 9 & 4 & 4 & 6 & 9 & 8 \\
\hline \multirow{3}{*}{$\begin{array}{l}\text { Groupe } 3 \\
\mathrm{n}=6\end{array}$} & $\mathrm{~m}$ & 10,33 & 6,20 & 0,745 & 3,25 & 59,43 & 16,81 & 2,06 & 0,0912 & 157 & 423 & 166 & 108 & 108 & 68 & 115 & 82 \\
\hline & $\sigma$ & 0,97 & 0,39 & 0,014 & 0,14 & 0,86 & 0,22 & 0,12 & 0,0172 & 4 & 48 & 15 & 7 & 6 & 10 & 3 & 9 \\
\hline & $\sigma \%$ & 9 & 6 & 2 & 4 & 1 & 1 & 6 & 19 & 3 & 11 & 9 & 6 & 6 & 15 & 2 & 11 \\
\hline \multirow{3}{*}{$\begin{array}{l}\text { Groupe } 4 \\
n=5\end{array}$} & $\mathrm{~m}$ & 12,90 & 8,19 & 0,682 & 2,55 & 56,94 & 15,20 & 2,53 & 0,1253 & 149 & 413 & 120 & 96 & 98 & 58 & 117 & 89 \\
\hline & $\sigma$ & 1,90 & 0,42 & 0,033 & 0,19 & 0,87 & 0,81 & 0,16 & 0,0107 & 8 & 37 & 9 & 5 & 7 & 9 & 4 & 13 \\
\hline & $\sigma \%$ & 15 & 5 & 5 & 8 & 2 & 5 & 6 & 9 & 5 & 9 & 8 & 6 & 7 & 16 & 3 & 15 \\
\hline \multirow{3}{*}{$\begin{array}{l}\text { Groupe } 5 \\
n=5\end{array}$} & $\mathrm{~m}$ & 16,10 & 5,38 & 0,652 & 2,93 & 56,46 & 14,78 & 2,46 & 0,1028 & 144 & 474 & 138 & 115 & 93 & 60 & 86 & 92 \\
\hline & $\sigma$ & 1,91 & 0,19 & 0,028 & 0,26 & 1,81 & 0,66 & 0,33 & 0,0114 & 18 & 41 & 16 & 11 & 6 & 3 & 10 & 10 \\
\hline & $\sigma \%$ & 12 & 4 & 4 & 9 & 3 & 4 & 13 & 11 & 13 & 9 & 11 & 10 & 7 & 5 & 12 & 11 \\
\hline
\end{tabular}

Tableau 2 : Moyenne $(\mathrm{m})$, écart-type $(\sigma)$ et écart-type réduit $(\sigma \%)$ des compositions chimiques des cinq groupes d'amphores gauloises d'Arles tels qu'ils apparaissent sur le graphique de la figure 4.

Table 2: Average chemical compositions $(m)$, standard deviation $(\sigma)$ and relative standard deviation ( $\sigma \%$ ) of the five Arles groups of amphorae as they appear on figure 4.

et enfin la marque [MI]P dans le groupe 4. Il ne semble donc pas exister de lien entre un groupe et une estampille ou ses différentes matrices.

Le lieu de découverte des amphores n'est pas non plus spécifique à un groupe. Dans le premier, les conteneurs proviennent du gisement $\mathrm{A}$, où était enfouit l'épave Arles-Rhône 3, hormis deux exemplaires découverts dans les dépotoirs de la place Jean-Baptiste Massillon (AMG817) et Arles-Rhône 1 (AMG841). Les amphores du groupe 2 sont issues du gisement A, hormis six amphores originaires du dépotoir ArlesRhône $1 / C N R$ et une de Brossolette. Le groupe 4 réuni quatre individus du gisement $\mathrm{A}$ et un du dépotoir Arles-Rhône 1/ $C N R$. Le groupe 3 rassemble quatre exemplaires mis au jour dans le gisement A, un dans le dépotoir Arles-Rhône 1/CNR et un au Cirque. Une amphore du groupe 5 a également été découverte sur ce site tandis que les quatre autres proviennent de la place Jean-Baptiste-Massillon.

Les cinq amphores constituant ce cinquième groupe sont datées entre le milieu du II $^{\mathrm{e}}$ siècle apr. J.-C. et le III ${ }^{\mathrm{e}}$ siècle apr. J.-C. Elles pourraient provenir d'un atelier qui n'était pas en activité lors de la formation des dépotoirs du gisement A et Arles-Rhône 1. La présence dans le groupe 1 d'une Gauloise 4, provenant également de la place Jean-BaptisteMassillon et datée de la seconde moitié du $\mathrm{II}^{\mathrm{e}}$ siècle apr. J.-C., témoigne de l'activité de cette officine jusqu'à cette période.

\section{Une origine locale d'amphores Gauloise 5, Fréjus-Lenzbourg et du timbre Q.C.H?}

\section{Amphores analysées par fluorescence $X$}

Lorigine locale des types Gauloise 5 (cinq exemplaires) (figure 5, $\left.\mathrm{n}^{\circ} 1-2\right)$ et Fréjus-Lenzbourg (trois exemplaires) (figure 5, $\mathrm{n}^{\circ} 3-4$ ), ainsi que d'une anse timbrée Q.C.H (figure $5, \mathrm{n}^{\circ} 5$ ), découverts dans le dépotoir du gisement Arles-Rhône 3, a été étudiée (tableau 3). L'analyse de ces deux modèles répondait à deux interrogations. La première était de déterminer si la zone de fabrication des Gauloise 5 pouvait s'étendre jusqu'à Arles. L'atelier de Sivier à Istres était jusqu'à présent le centre de production le plus occidental de cette amphore typiquement provençale (Marty, 2003; Mauné, 2013 : 354). La deuxième question était induite par la présence à Arles d'amphores à sauces de poissons Fréjus-Lenzbourg qui ne semblaient pas provenir de Fréjus. Les exemplaires forojuliens possèdent, en effet, un diamètre moyen à l'embouchure de $17,8 \mathrm{~cm}$ contre $13 \mathrm{~cm}$ pour ceux découverts à Arles (Brentchaloff, 1988: 181-182; Bigot, Djaoui, 2013 : 338). Par ailleurs, un réexamen macroscopique de la pâte de ces conteneurs a montré qu'elle semblait différente de celle des amphores de Fréjus, contrairement à ce que nous avions suggéré précédemment (ibid.). Nous voulions donc vérifier l'hypothèse d'une fabrication locale de ces amphores qui impliquerait un reconditionnement ou 


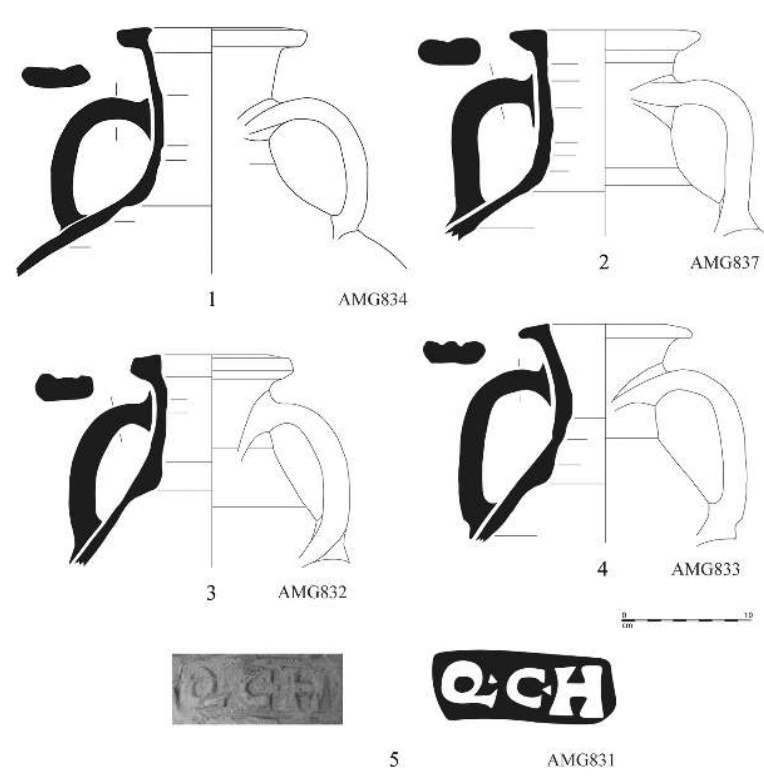

Figure 5 : (Voir planche couleur XI) Amphores Gauloise 5, FréjusLenzbourg et estampille Q.C.H analysées.

Figure 5: (See colour plate XI) Analysed Gauloise 5 and FréjusLenzbourg Amphorae and Amphorae with stamp Q.C.H.

une production de sauces et de salaisons de poissons près d'Arles. Pour cela leur composition chimique a été comparée à celle des groupes de référence d'Arles.

Enfin, l'analyse de l'amphore Gauloise 4 timbrée Q.C.H a été réalisée pour vérifier si cette estampille provient d'une officine rhodanienne. Sa pâte est effectivement comparable à celle d'amphores marquées bien représentées à Arles telles que A.P.M, MATVRI ou encore C.M.S (Laubenheimer, Schmitt, 2009, p. 129).

\section{Résultats des analyses}

La comparaison des compositions chimiques de ces amphores Gauloise 5, Fréjus-Lenzbourg et de l'anse timbrée Q.C.H a été effectuée par classification ascendante hiérarchique dans les mêmes conditions que précédemment (figure 6). On retrouve la répartition en 5 groupes dans un ordre légèrement différent, la présence de nombreux marginaux, et, à la fin du graphique, un groupe bien différencié. Ce groupe comprend des exemplaires à pâte sensiblement plus calcaire que le reste de l'échantillonnage. Il contient quatre des Gauloise 5 analysées, les Fréjus-Lenzbourg et deux amphores d'Arles déjà isolées pour leur taux de $\mathrm{CaO}$ très élevé. La majorité des amphores Gauloise 5 et FréjusLenzbourg analysées n'appartient donc pas aux groupes d'Arles.
Pour ce qui concerne les amphores Fréjus-Lenzbourg, les groupes de référence dont nous disposons pour Fréjus ne comportent pas d'individus à pâte si calcaire (Laubenheimer, Schmitt, 2009 : 104). S'il est donc assuré que ces conteneurs présentent des compositions différentes des productions arlésiennes, l'état actuel de nos connaissances des ateliers ne nous permet pas de proposer d'hypothèse d'origine. L'utilisation de l'amphore Fréjus-Lenzbourg, pour le conditionnement de sauces et salaisons de poissons, suggère plutôt une fabrication sur le littoral. En effet, les ateliers de Narbonnaise où sont façonnées des amphores à saumures se situent sur la côte ${ }^{11}$ ou au bord de zones lagunaires ${ }^{12}$.

Une seule des Gauloise 5 (AMG835) se classe dans le groupe 4 et pourrait correspondre à une production du groupe d'ateliers arlésiens. Jusqu'à présent, le centre potier le plus occidental fabriquant ce modèle était localisé à Istres, sur les rives de l'étang de Berre. Or, le groupe 4 se distingue bien de ce dernier (cf. infra). L'hypothèse d'une production de Gauloise 5 dans au moins un des ateliers de la région d'Arles est donc plausible. Il convient par ailleurs de rester circonspect à propos de l'absence de Gauloise 5 parmi les autres groupes 1, 2, 3 et 5 . En effet, peu d'individus de ce type ont été analysés et il s'agissait d'exemplaires dont la pâte possédait à l'œil nu des différences avec celles des Gauloise 4. Or, la majeure partie des Gauloise 5 découvertes dans les dépotoirs rhodaniens à Arles présente visuellement une pâte de type $\mathrm{A}$ ou $\mathrm{B}$. Elles pourraient donc correspondre à des amphores locales ${ }^{13}$.

Le timbre QCH (AMG831) se place seul en position marginale et ne peut donc être rattaché aux fabrications arlésiennes. Cette estampille proviendrait plutôt d'une zone de production, située près de la confluence entre la Durance et le Rhône. En effet, une concentration de cette marque est signalée dans ce secteur, à Caumont-sur-Durance (Vaucluse), où quinze exemplaires ont été découverts au lieu-dit SaintSymphorien (Mouraret, 2002-2003 : 106; Laubenheimer, Schmitt, 2009 : 124, fig. 83 et 125; Corbeel, Duperron,

11. En Narbonnaise, des Dr. 16 sont fabriquées à Cannes (Laubenheimer, 1989), des Gauloise 11 et Gauloise 16 ont été tournées à Mandelieu (Rivet, 1986 : 125-126, Laubenheimer, 1989 : 131-132; Lautier, Rothé, 2010 : 395), des Fréjus-Lenzbourg (atelier de SainteCroix), des Dr. 14, et des Dr. 16 ont été fabriquées à Fréjus (Laubenheimer et al. 1992, 19), et des Dr.7/11 ont été produites à Marseille (Bertucchi, 1992).

12. Des amphores Dr.7/11 ont été fabriquées dans les ateliers de la Plaine de Levreau (Laubenheimer, 1985 : 124) et de Bastide-neuve à Velaux (Mauné, Silvéréano, 2011 : 680-684), dans l'officine de La GrangeNeuve à Sigean (Laubenheimer ,1985: 117-120).

13. L'échantillonnage a privilégié cinq amphores qui se caractérisent par leur couleur grise. Les Gauloise 5 présentant cet aspect constituent une part importante du lot. Il était de ce fait intéressant de vérifier si elles provenaient d'une officine locale. 


\begin{tabular}{|c|c|c|c|c|}
\hline Numéro d'analyse & Site & Numéro d'Inventaire & Type & Timbre \\
\hline AMG831 & Arles-Rhône 3 & AR3-3007-152 & Gauloise 4 & Q.C.H \\
\hline AMG832 & Arles-Rhône 3 & AR3-2004-99 & Fréjus-Lenzbourg & - \\
\hline AMG833 & Arles-Rhône 3 & AR3-3018-264 & Fréjus-Lenzbourg & - \\
\hline AMG834 & Arles-Rhône 3 & AR3-3005-110 & Gauloise 5 & - \\
\hline AMG835 & Arles-Rhône 3 & AR3- C2-48 & Gauloise 5 & - \\
\hline AMG836 & Arles-Rhône 3 & AR3-3016-7 & Gauloise 5 & - \\
\hline AMG837 & Arles-Rhône 3 & AR3-2007-92 & Gauloise 5 & - \\
\hline AMG838 & Arles-Rhône 3 & AR3-2004-1 & Fréjus-Lenzbourg & - \\
\hline AMG863 & Arles-Rhône 3 & AR3-2031-165 & Gauloise 5 & - \\
\hline
\end{tabular}

Tableau 3 : Liste des amphores analysées par fluorescence X et mises au jour sur le site Gisement A/Arles-Rhône 3 pour établir ou non leur origine arlésienne.

Table 3: List of amphorae analyzed by XRF to establish or exclude their arlesian origin.

2013 : 401). Cette hypothèse n'a néanmoins pas pu être vérifiée par manque de références.

\section{Arles et les ateliers de la basse vallée du Rhône : étude comparative}

Afin d'attester l'existence de nouveaux ateliers nous avons confronté les résultats des analyses des amphores arlésiennes au réseau de références géochimiques des amphores gauloises de Narbonnaise (Laubenheimer, Schmitt, 2009). Une première comparaison (non ill.) à l'ensemble des ateliers de Gaule Narbonnaise a confirmé l'origine bas-rhodanienne des amphores découvertes à Arles. Nous avons donc confronté la composition chimique des pâtes des amphores des ateliers de la basse vallée du Rhône et localisés à proximité de cette région (Sivier à Istres, La Baume à Saint-Gilles-du-Gard, Les Tourelles et Privat à Beaucaire, Les Orgnes à Monfrin, Clausonne à Meynes, Village de Four à Sauveterre et la Treille à Saint-Laurent-des-Arbres) à celle de nos analyses ${ }^{14}$.

Cette comparaison a été effectuée par classification ascendante hiérarchique dans les mêmes conditions que précédemment (figure 7). On retrouve la répartition en cinq groupes des exemplaires d'Arles dans un ordre légèrement différent. Les ateliers de la basse vallée du Rhône sont bien individualisés et ne se mélangent pas aux groupes d'Arles. Seuls les exemplaires collectés à Saint-Gilles se dispersent mais nous avions déjà signalé la non pertinence de l'échantillonnage de ce site (Laubenheimer, Schmitt, 2009 : 81).

14. Nous ne disposons pas d'analyse permettant de connaitre la composition chimique de la pâte des amphores de Valescure à Beaucaire. Les cinq groupes identifiés à Arles présentent des compositions différentes de celles des deux ensembles de Font de Dringues à Vers-Pont-du-Gard (Laubenheimer, Schmitt, 2009 : 64).
Les marginaux d'Arles restent classés en position marginale et ne se rapprochent d'aucun des autres ateliers de la région.

Les groupes mis en évidence à Arles restent homogènes et ne se confondent pas avec les autres ateliers de la vallée du Rhône. Cela semble confirmer l'existence de cinq nouveaux ateliers amphoriques dans la région d'Arles.

\section{Conclusion}

Les analyses réalisées sur les amphores issues des dépotoirs d'Arles, révèlent l'existence de cinq nouvelles officines bas-rhodaniennes produisant des Gauloise 4 et, au moins pour l'une d'entre elles, des Gauloise 5. Ces résultats portent désormais à 14 le nombre de centres de production amphoriques dans la région d'Arles: "Proche Arles 1, 2, 3, 4 et 5 ", Clausonne à Meynes (Laubenheimer 1985 : 141-145), les Orgnes à Montfrin (Buffat, 2011 : 196), Font de Dringues à Vers-Pont-du-Gard (Ibid., n ${ }^{\circ} 133$ ), Village de Four à Sauveterre (Laubenheimer, 1985 : 159-161), La Treille à Saint-Laurent-des-Arbres (Laubenheimern 1985 : 156158), Privat, Le Mas des Tourelles et Valescure à Beaucaire (Provost et al., 1999 : 225; Laubenheimer, 1985: 131-135; 1989 : 111 ; Ignace, 2001, 2002, 2003, 2004) et le Mas de la Baume à Saint-Gilles (Laubenheimer, 1985 : 152-155). Ce nombre reste probablement sous-estimé, au regard de l'importance de la diffusion des amphores de cette région vers les centres de consommation du limes germanique, de Bretagne et de Méditerranée. Cependant, il reste à localiser précisément les cinq ateliers mis en évidence par nos analyses. Ils pourraient correspondre à des centres de productions situés dans les quartiers péri-urbains d'Arles, comme à Fréjus (Excoffon 2009 : 15-18), ou dans la basse vallée du 


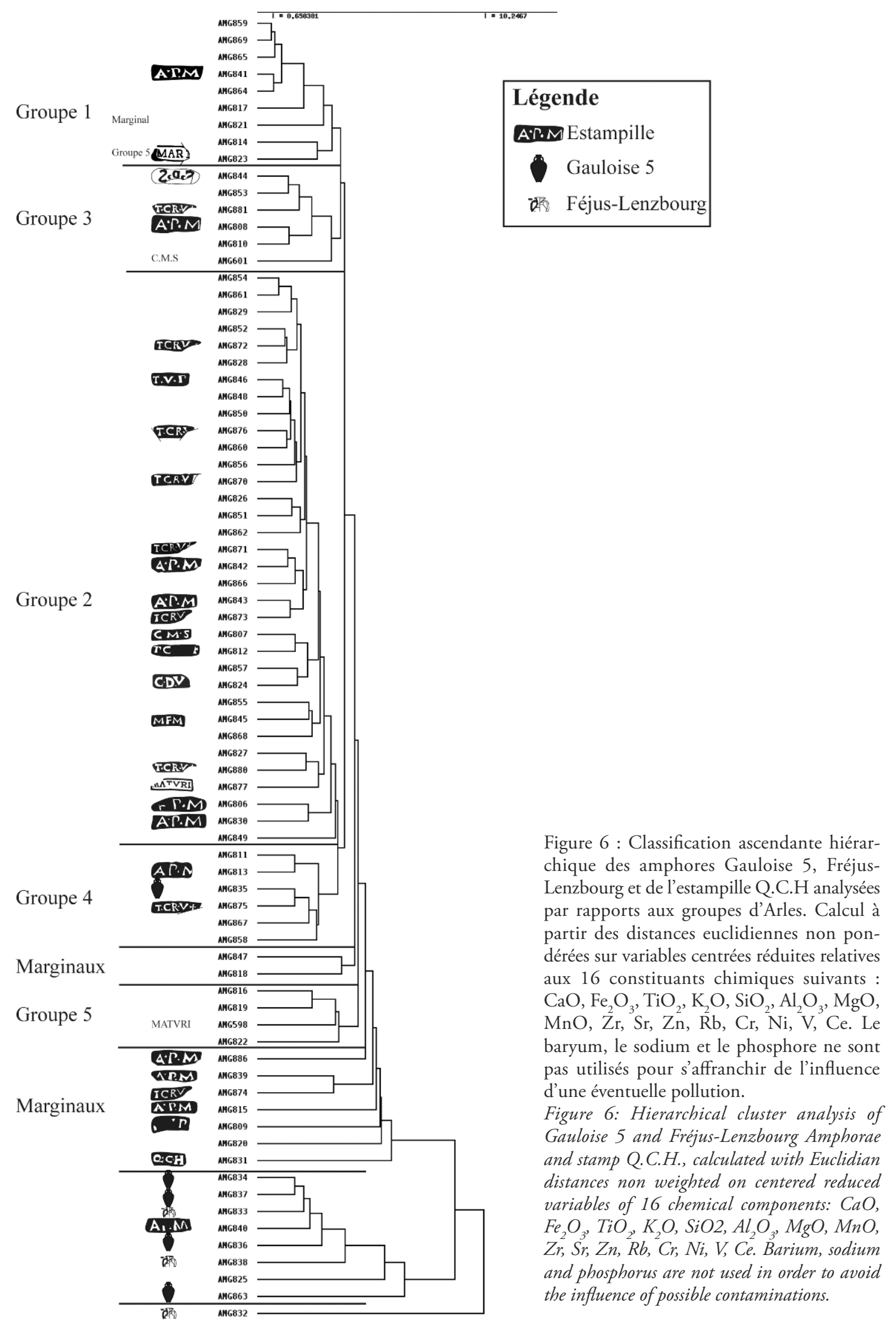

ArCheoSCIEnCes, revue d'archéométrie, 41(1), 2017, p. 83-99 


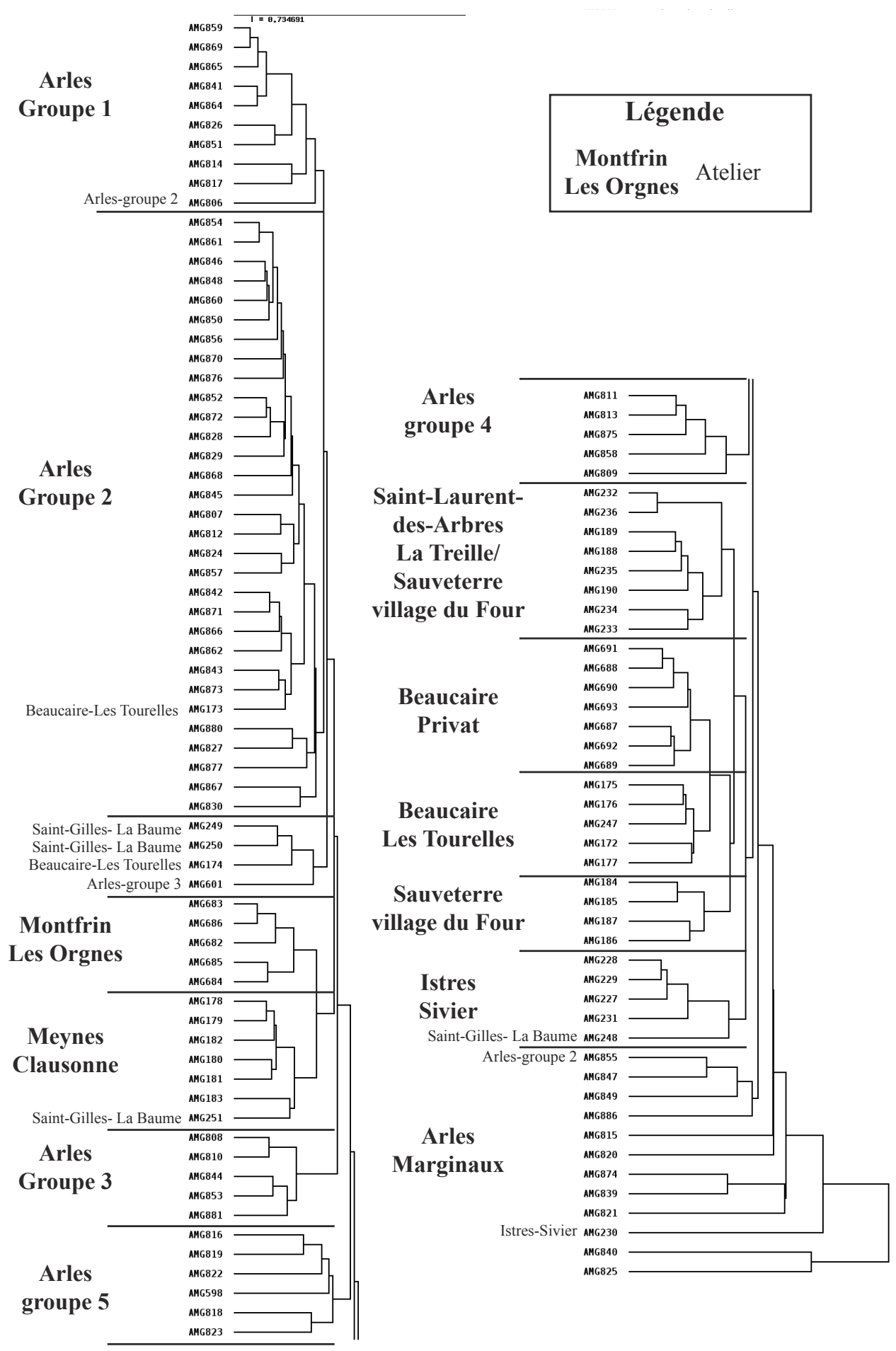

Figure 7 : Classification ascendante hiérarchique des groupes mis en évidence à Arles par rapport aux ateliers de la Basse vallée du Rhône. Calcul à partir des distances euclidiennes non pondérées sur variables centrées réduites relatives aux 16 constituants chimiques suivants : $\mathrm{CaO}, \mathrm{Fe}_{2} \mathrm{O}_{3}, \mathrm{TiO}_{2}, \mathrm{~K}_{2} \mathrm{O}, \mathrm{SiO}_{2}, \mathrm{Al}_{2} \mathrm{O}_{3}, \mathrm{MgO}, \mathrm{MnO}, \mathrm{Zr}, \mathrm{Sr}, \mathrm{Zn}, \mathrm{Rb}, \mathrm{Cr}, \mathrm{Ni}, \mathrm{V}$, Ce. Le baryum, le sodium et le phosphore ne sont pas utilisés pour s'affranchir de l'influence d'une éventuelle pollution.

Figure 7: Hierarchical cluster analysis of Arles groups relatives to known workshops in the lower Rhône valley. Calculated with Euclidian distances non weighted on centered reduced variables of 16 chemical components : $\mathrm{CaO}, \mathrm{Fe}_{2} \mathrm{O}_{3}, \mathrm{TiO}_{2}, \mathrm{~K}_{2} \mathrm{O}, \mathrm{SiO}_{2}, \mathrm{Al}_{2} \mathrm{O}_{3}, \mathrm{MgO}, \mathrm{MnO}, \mathrm{Zr}, \mathrm{Sr}, \mathrm{Zn}, \mathrm{Rb}, \mathrm{Cr}$, $\mathrm{Ni}, \mathrm{V}, \mathrm{Ce}$. Barium, sodium and phosphorus are not used in order to avoid the influence of possible contaminations. 
Rhône. Le Rhône facilite en effet l'acheminement jusqu’à la zone portuaire de Trinquetaille des amphores façonnées dans un rayon d'une trentaine de kilomètres autour de l'agglomération.

\section{Les indices d'ateliers amphoriques}

Les fouilles et les prospections menées dans la région d'Arles ont révélé l'existence d'une quinzaine d'ateliers qui pourraient avoir fabriqué des amphores, bien qu'ils n'aient pas tous livré des rebuts de cuisson (figure 8$)^{15}$. Plusieurs indices probants attestent l'existence d'un ou plusieurs ateliers dans le quartier de Trinquetaille. En premier lieu, l'abondance des marques dans les groupes "Proche-Arles" suggère l'existence de nombreuses officines. De plus, des zones de rebuts de production ont été repérées en plusieurs points de la ville (figure 1). Ainsi, les gisements A et ArlesRhône 1 sont distants de $500 \mathrm{~m}$, tandis qu'une concentration de mobilier est également signalée dans le secteur de la Pointe sur la presqu'île des Sables (Rothé, Heijmans, 2008 : 611). Enfin, une officine amphorique a été identifiée à l'angle nord-ouest du cimetière de Trinquetaille. Elle se manifeste par la présence " d'une couche très épaisse d'argile avec briques crues renfermant une grande masse de débris provenant d'un four de portiers, avec anses et fragments d'amphores soudés ensemble par le feu et vitrification " (Benoit 1938; Rothé, Heijmans, 2008 : 637). Sur la rive droite du Rhône, plusieurs ateliers antiques sont mentionnés comme "four(s) de tuiliers " dans la bibliographie: les centres potiers de la route de Nîmes et de la route de Beaucaire à Saint-Gilles du Gard (Laubenheimer, 1985 : 152), de la Vaque (Provost et al., 1999 : 236) et du Mas de Broussan à Bellegarde (Buffat, 2011 : 189). Signalons également le site d'Espeyran (Saint-Gilles-du-Gard), où les opérations archéologiques ont permis de mettre en évidence un vaste atelier constitué d'au moins une douzaine de fours (Christol et al., 2011 : 148-149) ${ }^{16}$. Sur la rive gauche du Rhône, l'officine de la Taulière (Paradou) a été identifiée à partir de la découverte de tegulae crues (Gateau, Gazenbeek, 1999 : 244). Au sud-est, trois ateliers ont été repérés sur la commune de Saint-Martin-du Crau. Le premier est localisé à environ $500 \mathrm{~m}$ du Rhône, au Petit Abondoux, et les deux autres, Vergieres 3 et Baussenq 8 à environ huit et neuf kilomètres à l'est de ce dernier (Excoffon, 2009 : 18).

15. La caractérisation physico-chimiques des productions de ces ateliers demeure inconnue car nous n'avons pas eu accès au mobilier issu de ces sites dans le cadre de notre recherche.

16. La fouille exhaustive de l'un d'entre eux n'a pas livré d'amphores mais fournit plusieurs indices militant en faveur de leur production dès l'époque augustéenne (Bigot 2015).
Enfin, entre la confluence du Rhône et de la Durance, trois ateliers ont été installés autour du massif de la Montagnette. L'officine de l'ancienne décharge municipale à Barbentane a été identifiée grâce à la découverte de " fours à potiers de différentes dimensions" (Gateau, Gazenbeek, 1999 : 113; Excoffon, 2009 : 15), celle des Bouisses (Boulbon) par la mise au jour d'argile cuite et de tuiles surcuites (Ibid. : $132)^{17}$ et celle du Mas de la Sour Peyronne (Tarascon) par la découverte de tuiles surcuites (Ibid. : 410) (figure 8).

Ces ateliers présentent une situation avantageuse pour développer une production d'amphores. En effet, ils sont localisés à proximité d'affleurements d'argiles et à côté du Rhône qui est susceptible de permettre à la fois un approvisionnement en matières premières, nécessaires à la fabrication des amphores (eau, argile et bois ${ }^{18}$ ) et une diffusion aisée de ces produits pondéreux, via le Rhône ${ }^{19}$. Les ateliers mis en évidence par les analyses physico-chimiques à Arles peuvent donc correspondre à ces sites.

\section{Chronologie des ateliers}

Les amphores analysées pour cette étude sont datées entre la fin du $\mathrm{I}^{\mathrm{er}}$ siècle apr. J.-C. et le milieu du $\mathrm{II}^{\mathrm{e}}$ siècle apr. J.-C., hormis une douzaine de Gauloise 4, de la seconde moitié du II $^{\mathrm{e}}$ siècle apr. J.-C. et du III ${ }^{\mathrm{e}}$ siècle apr. J.-C. On ignore si les cinq ateliers de la basse vallée du Rhône produisant ces contenants fonctionnaient dès la période julio-claudienne et combien étaient encore en activité entre le milieu du $\mathrm{II}^{\mathrm{e}} \mathrm{s}$. et le $\mathrm{IV}^{\mathrm{e}}$ siècle. Plusieurs indices semblent témoigner du dynamisme de cette région durant toute la période de production des amphores gauloises. Ainsi, une fouille récente à Espeyran suggère une production de celles-ci dès le dernier tiers du $\mathrm{I}^{\mathrm{er}}$ siècle av. J.-C. (Bigot, 2015). La période julio-claudienne demeure néanmoins profondément méconnue, puisque les niveaux les plus anciens atteints dans les dépotoirs du Rhône à Arles datent des années 60 apr. J.-C. (Bigot, Djaoui, 2013 : 390). En revanche, les fouilles de L. Long ont livré des quantités importantes d'amphores gauloises qui semblent attester

17. Par ailleurs, des amphores gauloises ont également été découvertes lors de prospections sur ce site (Gateau, Gazenbeek, 1999 : 244).

18. Sur le Rhône, cette activité est attestée pour l'Antiquité par un negotiator artis ratiariae (CIL XIII, 2035), qui serait, selon A. Grenier, un commerçant en bois transporté jusqu'à Lyon par flottage (Grenier, $1934: 543)$.

19. En effet, comme le souligne A. Tchernia, «si le coût d'un transport par mer est de 1, celui du trajet fluvial est de 4,7 et la voie de terre de 22,6» (2011 : 127). C’est pourquoi le transport fluvial était généralement préféré au transport terrestre et largement prépondérant, notamment pour les marchandises pondéreuses ou encombrantes (Christol, Fiches, 1999 : 150). Or, l'importance de la voie rhodanienne était telle qu'on a pu affirmer qu'elle était empruntée par «le plus important réseau d'échanges du monde romain " (Ibid. : 141). 


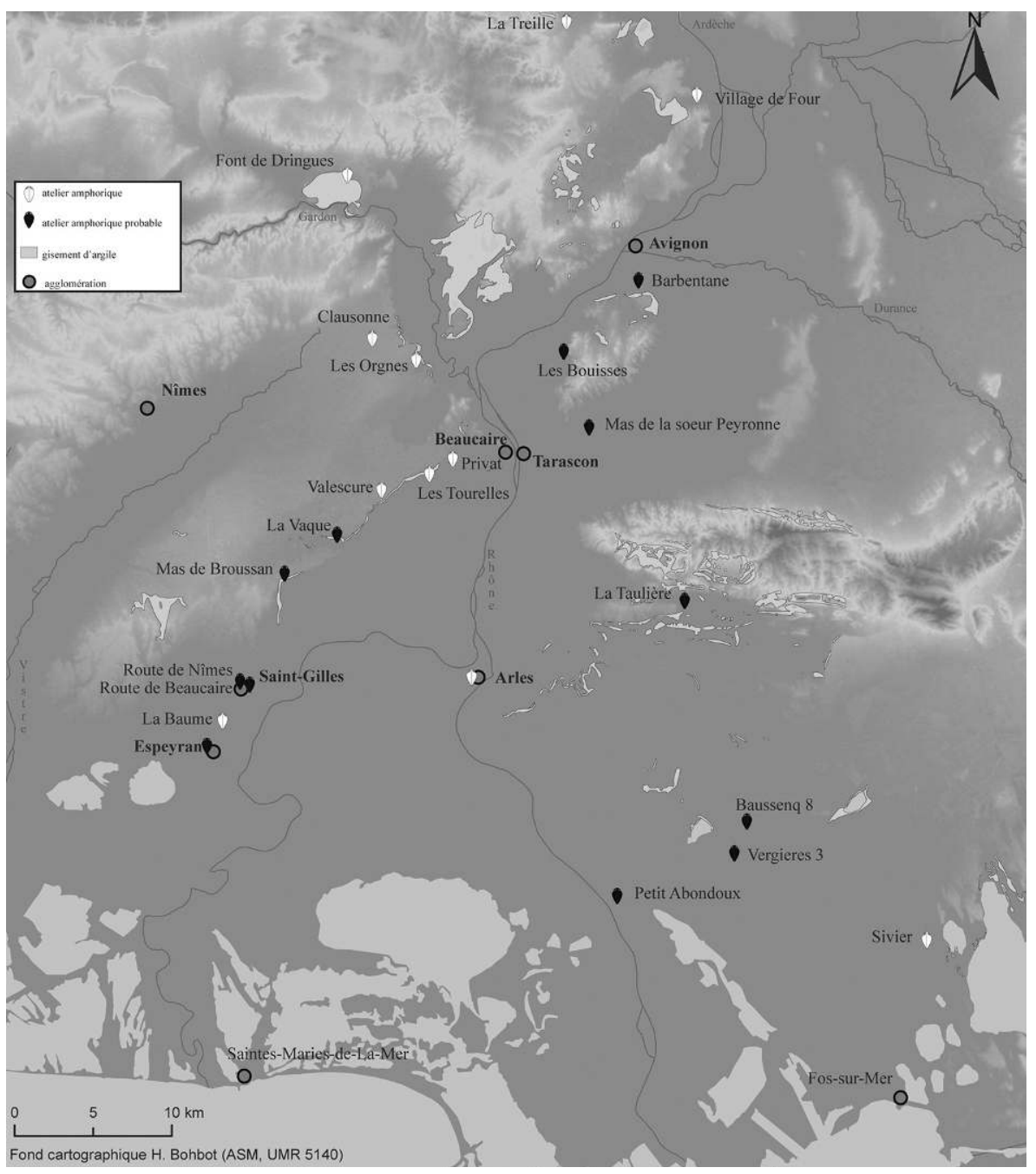

Figure $8:$ (Voir planche couleur XII) Localisation des ateliers de la basse vallée du Rhône et des principaux gisements d'argiles les jouxtant (DAO F. Bigot; fonds cartographiques $\mathrm{H}$. Bohbot, [http://infoterre.brgm.fr] et [http://www.georisques.gouv.fr]]).

Figure 8: (See colour plate XII) Location of the workshops in the lower Rhône valley and the main nearby clay deposits (DAO F. Bigot; cartographic frame $H$. Bohbot, [http://infoterre.brgm.fr] et [http:// www.georisques.gouv.fr/]). leur production dans la région d'Arles jusqu'au milieu du IV $^{\mathrm{e}}$ siècle (Long, Duperron, 2011 ; Long, Duperron, 2013 ; Long, Duperron, 2014). Cette hypothèse est étayée par la découverte d'estampilles, dont on ignore le lieu de production, dans des niveaux du $\mathrm{III}^{\mathrm{e}}$ siècle (L.SE, MERCATOR) et du Iv ${ }^{e}$ siècle (ALBIN) (Corbeel, Duperron, 2013 : 426). De plus, l'analyse du timbre MARTIAL (AMG823) atteste l'existence d'une officine de la région d'Arles apposant cette marque durant le $\mathrm{III}^{\mathrm{e}}$ siècle. On peut donc supposer la création durant la seconde moitié du $\mathrm{II}^{\mathrm{e}}$ siècle de nouvelles officines dont l'activité perdure au III $^{\mathrm{e}}$ siècle et vraisemblablement jusqu'au Iv ${ }^{e}$ siècle. Cependant, aucun atelier de ces périodes n’a encore été fouillé. 
Composition chimique des amphores analysées

Chemical composition of analysed amphorae

\begin{tabular}{|c|c|c|c|c|c|c|c|c|c|c|c|c|c|c|c|c|c|c|c|c|c|c|c|c|}
\hline & $\%$ & $\%$ & $\%$ & $\%$ & $\%$ & $\%$ & $\%$ & $\%$ & $\%$ & $\%$ & $\mathrm{ppm}$ & $\mathrm{ppm}$ & $\mathrm{ppm}$ & $\mathrm{ppm}$ & $\mathrm{pm}$ & ppm & ppm & ppm & \begin{tabular}{|l|} 
ppm \\
\end{tabular} & ppm & $\mathrm{ppm}$ & ppm & ppm & $\mathrm{ppm}$ \\
\hline $\mathrm{m}$ & $\mathrm{OO}$ & ${ }_{2} \mathrm{O}_{3}$ & $\mathrm{O}_{2}$ & $\mathrm{~K}_{2} \mathrm{O}$ & $\mathrm{SiO}_{2}$ & $\mathrm{ll}_{2} \mathrm{O}_{3}$ & $\mathrm{MgO}$ & $\mathrm{MnO}$ & $\mathrm{Na}_{2} \mathrm{O}$ & $\mathrm{P}_{2} \mathrm{O}_{5}$ & $\mathrm{Zr}$ & Sr & $\mathrm{Rb}$ & $\mathrm{Zn}$ & $\mathrm{Cr}$ & $\mathrm{Ni}$ & $\mathrm{La}$ & $\mathrm{Ba}$ & V & $\mathrm{Ce}$ & $\mathrm{Y}$ & Th & $\mathrm{Pb}$ & $\mathrm{Cu}$ \\
\hline MG5 &, 59 & 26 & 646 & 2,50 & 5,08 & 4,58 & 37 & \begin{tabular}{|l|l|l|l|} 
\\
\end{tabular} & 2.7 & 32 & 171 & 531 & 111 & 120 & 99 & 62 & & 450 & 88 & 84 & & & & \\
\hline MG601 & & 5 & & 3,08 & 20 & & & & & & 158 & 14 & 144 & 96 & 15 & 86 & & 33 & 17 & 77 & & & & \\
\hline GG806 & 98 & 6 & & 3,08 & & & 68 & 275 & & 20 & 120 & & 158 & 106 & 03 & 5 & & 05 & 28 & of & 23 & 12 & 27 & 27 \\
\hline IG807 & 16 & & & 2,9 & & & 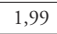 & 1085 & & & 138 & & 155 & 100 & 00 & 60 & & 555 & 15 & 87 & 25 & & 25 & 35 \\
\hline GG808 & & 5,9 & 80 & 3,31 & 72 & 88 & 80 & 0841 & 0,6 & 17 & 15 & 431 & 73 & 10 & 97 & 59 & & 26 & 16 & 88 & 30 & 10 & 39 & 33 \\
\hline MG809 & 93 & 7,5 & 555 & 2,2 & 39 & 99 & 2 & 1203 & 0,4 &, 21 & 161 & & 08 & 87 & 8 & 51 & & 62 & 16 & 109 & 26 & 10 & 15 & 21 \\
\hline MG810 & 30 & 6,0 & 756 & 3,31 &, 52 & 7,07 & 1,92 & 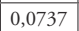 & 0,65 & 0,16 & 159 & 377 & 178 & 108 & 104 & 59 & & 519 & 114 & 97 & 26 & 11 & 35 & 30 \\
\hline MG811 & 06 & 8,3 & 772 & 2,58 & 5,27 & 5,40 & 2,72 & 0,1266 & 0,44 & 0,17 & 144 & 419 & 116 & 97 & 97 & 48 & & 527 & 118 & 89 & 24 & 11 & 19 & 26 \\
\hline MG81 & 15,77 & 5,9 & 668 & 2,63 & 56, & 39 & 2,3 & 0,1 & 0,4 & & 138 & 4 & 136 & 100 & 97 & 5 & & 454 & 131 & 88 & & 12 & 30 & 31 \\
\hline 4G813 & 70 & 8,03 & & 2,73 & 92 & 38 & 2,5 & 1246 & 0 & 17 & 142 & 01 & 132 & 101 & 99 & 61 & & \begin{tabular}{|l|l|}
587 \\
\end{tabular} & 111 & 94 & 26 & & 24 & 24 \\
\hline 8814 & 86 & & & 3,4 & & & & | & & & 12 & & 170 & 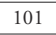 & 106 & 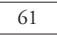 & & \begin{tabular}{|l|l|}
721 \\
\end{tabular} & & 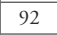 & 4 & & & 37 \\
\hline GG81 & & & & 2,8 & 92 & 5,36 & 2,6 & 038 & 0,3 & 2 & 120 & & 140 & 112 & 107 & 6. & 4 & \begin{tabular}{|l|l|}
1101 \\
\end{tabular} & 117 & 70 & 24 & & 22 & 30 \\
\hline MG816 & 00 & 5,35 & 40 & 2,97 & 46 & 01 & 2,07 & 1129 & 0,6 & 0,60 & 154 & 422 & 142 & 106 & 86 & 62 & 2 & 440 & 75 & 106 & 27 & 10 & 26 & 40 \\
\hline MG817 & 87 & 6,13 & 06 & 3,68 & 6,63 & 91 & 2,57 & \begin{tabular}{|l|l|}
0,0896 \\
\end{tabular} & 0,77 & 0,44 & 12 & 20 & 183 & 107 & 113 & 61 & & 33 & 101 & 4 & 26 & 14 & 75 & 47 \\
\hline MG818 & & 5, & & 3,29 & 55,50 & & 3,3 & & & & 16 & 2 & 151 & 1 & 100 & 61 & & 483 & 1 & & 28 & & & so \\
\hline MG81 & 846 & 5,5 & 680 & 2,9 & 54,72 &, 30 & 2,3 & 1080 & & 0,3 & 13 & 462 & 147 & . & 89 & 55 & & 98 & 8 & & 26 & 12 & (5) & 41 \\
\hline GG820 & 13,89 & 5,1 & 0,657 & 3,2 & 59,11 & 14,53 & 1,9 & {$[0,0031]$} & 0,4 & 0,82 & 14 & 490 & 155 & 95 & (11 & 54 & & 53 & & 2 & 2) & & 22 & 66 \\
\hline G8821 & ,24 & 6,3 & 684 & 3,8 &, 15 & , & & 0,1132 & & & 10 & & 1 & & 111 & & & 741 & & & 4 & & & 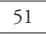 \\
\hline MG822 & 82 & 5,1 & & 3,1 & 35 & 40 & & 090 & & & 12 & & 14 & 1 & 92 & 6 & & 508 & & & 25 & & 48 & 55 \\
\hline MG823 & 65 & 5,6 & 80 & 3,05 & 67 & 15,61 & 2,6 & 0905 & 0,3 & 0,51 & 133 & 495 & 151 & 13 & 100 & 61 & & 443 & 101 & 92 & 30 & & 46 & 65 \\
\hline MG824 & 12 & 5,3 & 57 & 2,70 & 79 & 15,25 & & 0867 & & 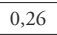 & 13 & 458 & 141 & 94 & 81 & 58 & & 433 & 1 & & 28 & & 74 & 33 \\
\hline MG8 & & 4.6 & & 2,25 & & & & & & & 1 & 6 & 116 & 1 & 75 & 5 & 2 & 319 & & 69 & 24 & 8 & 42 & . \\
\hline AG826 & & 5,9 & 689 & & 50 & 98 & & 1162 & 0 , & 0,1 & 14 & 501 & 164 & 102 & 10 & 69 & 2 & 418 & 118 & 74 & 28 & 12 & 27 & 32 \\
\hline IG827 & 27 & 7,1 & 682 & & 52 & & & 15 & & & 14 & 88 & 111 & 9 & 101 & & & 811 & & & 28 & & 24 & 30 \\
\hline UG828 & 6,26 & 6,3 & $0 / 4$ & 2,8 & בל, & 26 & & 0,1290 & & & 1 & 4 & 139 & 9 & 101 & & & 476 & & & 7 & & 0 & 31 \\
\hline MG829 & 98 & 5,6 & 65 & & ,46 & 14 & & 1114 & & & 14 & & 147 & 9 & 98 & & & 429 & & & 27 & & 25 & 27 \\
\hline MG830 & 66 & 7,22 & 557 & 2,65 & 39 & 5,54 & & 11 & & 0,21 & 12 & 511 & 122 & 93 & 107 & & & 592 & & & 5 & & 1 & 25 \\
\hline MG831 & & & & & & & & 0,1222 & & & 1 & 70 & 99 & 91 & 188 & 6 & & 463 & & & 2 & & 20 & 29 \\
\hline MG8 & & 5,1 & & 3,37 & & & & & & & 2 & 258 & 114 & 34 & 6 & 3 & 3 & 906 & & 19 & 2 & 11 & 11 & 22 \\
\hline $\mathrm{G} 8$ & & 5,2 & & 1,80 & 5,04 & 23 & & 0,092 & & 0 , & 16 & 490 & 83 & 6 & 6 & 3 & 3 & 499 & & & 27 & & 11 & 22 \\
\hline MG834 & 51 & 5,6 & & 1,7 & 411 & & & 10 & & & 1 & 494 & 10 & & & & & 43 & & & 28 & & & 23 \\
\hline MG835 & 42 & 7,7 & & 2, & & 68 & & 0,116 & & & 1 & & 11 & & 103 & & & 782 & & 72 & 26 & 14 & 19 & 25 \\
\hline MG836 & 65 & 4,8 & 503 & 1, &, 56 & 45 & & 0,0798 & & & 1 & 465 & 10 & & 6 & & & 465 & & & & & & 24 \\
\hline MG837 & & & & & & & & 0,11 & & & 16 & & & & 6 & & & 23 & & & & & 2 & 24 \\
\hline , & & & & & & & & & & & 1 & 5 & 5 & 65 & 58 & 3 & & 3 & & 72 & 2 & 8 & 16 & 22 \\
\hline 80 & & & & & & & & 0 & & 0 , & 12 & 09 & 168 & 114 & 10 & 78 & 4 & 701 & 1 & 79 & 28 & 15 & 79 & 36 \\
\hline 4G840 & 42 & 4,6 & & & 190 & 2 & & 0,128 & & 0,1 & 10 & 67 & 92 & 6 & 56 & & & 369 & & & 27 & & & 21 \\
\hline MG841 & ,67 & 6,8 & & 3,2 & 55.38 & 0 & & 0,1175 & & 0, & 125 & 487 & 160 & 108 & 115 & 68 & & 775 & 115 & 72 & 24 & 11 & 24 & 31 \\
\hline MG842 & 22 & & & & & & & 0,097 & & & 16 & & 15 & & 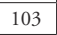 & & & 586 & & & 26 & & 22 & 24 \\
\hline MG843 & 94 & & 699 & & 18 & & & 13 & & & 19 & 4 & 154 & 1 & 105 & & & 459 & 19 & & 27 & & 1 & 26 \\
\hline 1G844 & & & & & & & & & & & 1 & & 174 & & 11 & & & 16 & & & 9 & 14 & 27 & 31 \\
\hline 38 & & & & & & & & & & & 1 & 46 & 134 & 88 & 99 & 57 & & 366 & & & 8 & 77 & 13 & 24 \\
\hline $4 \mathrm{G} 846$ & & & & & 56, & & & & & & 1 & 4 & 13 & 98 & 100 & 68 & & 433 & & & 29 & & 21 & 30 \\
\hline AG847 & & & & & 53,0 & & & 0,14 & & 0, & 16 & 499 & 12 & 91 & 107 & 5 & & 517 & & 67 & 28 & 1 & 12 & 19 \\
\hline MG848 &, 55 & & & & 55,54 & 15,6 & & 0,11 & & 0 , & 13 & 490 & 15 & 11 & 106 & 66 & & 418 & & 70 & 28 & 12 & 20 & 32 \\
\hline MG849 & 78 & & & & 53,98 & & & 138 & & 0 , & 13 & 520 & 13 & 87 & 96 & & & 436 & & & 25 & & 17 & 25 \\
\hline 4G850 & 90 & & & & & & & & & 0 & 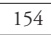 & 530 & 138 & 97 & 102 & 67 & & 557 & 05 & 80 & 28 & 1 & 21 & 29 \\
\hline MG851 & & & & & & & & & & & $T$ & 02 & 155 & 101 & 116 & & 4 & 641 & & 67 & 7 & & 18 & 29 \\
\hline 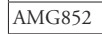 & & & & & & & & & & 0 , & 1 & 41 & 141 & $9 ?$ & 109 & 0. & 3 & 484 & & 67 & 27 & 1 & 9 & 29 \\
\hline & & & & & & & & & & & & & 17 & 10 & 110 & 66 & & 552 & & & & & 26 & 29 \\
\hline$\overline{G 8}$ & & & & & & & & & & & 1 & & & & & & & & & & 9 & & 17 & 30 \\
\hline MG855 & & & & & & & & & & 0 , & 18 & 541 & 13 & 93 & 11 & 66 & 3 & 409 & & 70 & 88 & 1 & 15 & 30 \\
\hline MG856 & & & & & & & & (c) & & 0 , & 152 & 442 & 13 & 99 & 104 & 68 & & 486 & 09 & 83 & 28 & 1 & 23 & 31 \\
\hline MG857 & & & & & & & & & & 0 , & 1 & 49 & 141 & 94 & 100 & 59 & 4 & 13 & 05 & 74 & 26 & 10 & 15 & 28 \\
\hline MG858 & & & & 2,47 & & & & & 0 , & 0 & 15 & 37 & 20 & 99 & 107 & 71 & 3 & 502 & & 81 & 29 & 13 & 23 & 32 \\
\hline 8859 & & & & & & & & & 0, & 0 , & 1 & 50 & 176 & 11 & 110 & 0 & 3 & 27 & & 7 & 7 & & 24 & 30 \\
\hline 6860 & & & & & & & & & & 0 & 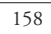 & 48 & 1 & 99 & 102 & 64 & 3 & 441 & & 78 & 9 & 11 & 18 & 31 \\
\hline G8 & & & & & & & & & & & & & & & 16 & & & 8 & & & 8 & & 3 & 32 \\
\hline 1G8 & & & & & & & & & & & & & 155 & 1 & 107 & 68 & & 5 & 12 & 68 & 30 & 11 & 19 & 30 \\
\hline MG863 & & & & & & & & & & & & 930 & 50 & 62 & 47 & 34 & 3 & 465 & 65 & 63 & 25 & 4 & 4 & 21 \\
\hline MG864 & 76 & 6, & 0,694 & 3,30 & 54 & 16, & 2,6 & 0,1149 & 0,60 & 0,19 & 127 & 490 & 167 & \begin{tabular}{|l|}
109 \\
\end{tabular} & 107 & 69 & 44 & 548 & 115 & 72 & 27 & 12 & 26 & 31 \\
\hline MG865 & 13 & & 0 & 3,41 & 54, & 17 , & 2,81 & 0976 & 0,71 & 0, & 128 & \begin{tabular}{|l|l|}
479 \\
\end{tabular} & 178 & 11 & 114 & 71 & 36 & 534 & 112 & 73 & 27 & 11 & 29 & 31 \\
\hline MG866 & & 5,8 & 0,7 & 2,6 & ני & 15 & & & 0,3 & , & 10 & 45 & 130 & 10 & 10 & 68 & 4 & 95 & & 73 & 29 & 1 & 24 & 30 \\
\hline G867 & 13,49 & 7,04 & & 2,25 & (1) & & & $x+5$ & 0,33 & 0,17 & 158 & 529 & 106 & 97 & 108 & 65 & 38 & 502 & 123 & 73 & 29 & 10 & 21 & 30 \\
\hline
\end{tabular}




\begin{tabular}{|c|c|c|c|c|c|c|c|c|c|c|c|c|c|c|c|c|c|c|c|c|c|c|c|c|}
\hline & $\%$ & $\%$ & $\%$ & $\%$ & $\%$ & $\%$ & $\%$ & $\%$ & $\%$ & $\%$ & ppm & ppm & ppm & ppm & ppm & $\mathrm{ppm}$ & ppm & ppm & $\mathrm{ppm}$ & ppm & ppm & $\mathrm{ppm}$ & ppm & ppm \\
\hline & $\mathrm{CaO}$ & $\mathrm{Fe}_{2} \mathrm{O}_{3}$ & $\mathrm{TiO}_{2}$ & $\mathrm{~K}_{2} \mathrm{O}$ & $\mathrm{SiO}_{2}$ & $\mathrm{Al}_{2} \mathrm{O}_{3}$ & $\mathrm{MgO}$ & $\mathrm{MnO}$ & $\mathrm{Na}_{2} \mathrm{O}$ & $\mathrm{P}_{2} \mathrm{O}_{5}$ & $\mathrm{Zr}$ & $\mathrm{Sr}$ & $\mathrm{Rb}$ & $\mathrm{Zn}$ & $\mathrm{Cr}$ & $\mathrm{Ni}$ & $\mathrm{La}$ & $\mathrm{Ba}$ & $\mathrm{V}$ & $\mathrm{Ce}$ & $\mathrm{Y}$ & Th & $\mathrm{Pb}$ & $\mathrm{Cu}$ \\
\hline AMG868 & 16,49 & 5,56 & 0,711 & 2,69 & 56,18 & 15,32 & 2,09 & 0,1130 & 0,43 & 0,23 & 175 & 537 & 137 & 99 & 104 & 68 & 31 & 436 & 110 & 81 & 32 & 12 & 19 & 29 \\
\hline AMG869 & 14,67 & 6,45 & 0,696 & 3,37 & 53,91 & 17,02 & 2,70 & 0,1087 & 0,67 & 0,20 & 127 & 500 & 174 & 111 & 110 & 70 & 41 & 527 & 111 & 74 & 27 & 12 & 30 & 30 \\
\hline AMG870 & 13,96 & 5,86 & 0,718 & 2,57 & 57,48 & 16,02 & 2,28 & 0,1119 & 0,64 & 0,18 & 150 & 480 & 127 & 102 & 107 & 68 & 35 & 531 & 99 & 73 & 26 & 12 & 22 & 31 \\
\hline AMG871 & 11,74 & 6,01 & 0,701 & 2,88 & 58,45 & 15,67 & 2,73 & 0,0999 & 1,35 & 0,17 & 149 & 461 & 136 & 101 & 106 & 68 & 33 & 546 & 106 & 74 & 27 & 14 & 10 & 27 \\
\hline AMG872 & 14,86 & 7,14 & 0,668 & 2,75 & 56,06 & 15,19 & 2,16 & 0,1284 & 0,66 & 0,20 & 146 & 489 & 140 & 97 & 104 & 63 & 41 & 527 & 113 & 70 & 25 & 10 & 18 & 30 \\
\hline AMG873 & 11,92 & 5,93 & 0,706 & 3,02 & 58,52 & 15,86 & 2,64 & 0,1567 & 0,87 & 0,19 & 149 & 434 & 143 & 106 & 104 & 67 & 42 & 515 & 109 & 72 & 28 & 10 & 20 & 31 \\
\hline AMG874 & 10,85 & 6,22 & 0,727 & 2,72 & 59,69 & 16,23 & 2,35 & 0,2075 & 0,56 & 0,23 & 160 & 418 & 135 & 106 & 135 & 83 & 41 & 697 & 105 & 81 & 28 & 17 & 32 & 32 \\
\hline AMG875 & 10,82 & 8,47 & 0,667 & 2,71 & 58,42 & 15,01 & 2,62 & 0,1129 & 0,79 & 0,20 & 145 & 474 & 125 & 98 & 100 & 59 & 39 & 378 & 118 & 74 & 25 & 12 & 28 & 30 \\
\hline AMG876 & 15,89 & 5,77 & 0,673 & 2,20 & 56,69 & 15,14 & 2,35 & 0,1143 & 0,79 & 0,19 & 151 & 474 & 143 & 99 & 99 & 61 & 39 & 609 & 106 & 75 & 26 & 8 & 16 & 28 \\
\hline \begin{tabular}{|l|} 
AMG877 \\
\end{tabular} & 15,96 & 5,62 & 0,697 & 2,37 & 56,40 & 15,72 & 2,33 & 0,1883 & 0,18 & 0,33 & 149 & 466 & 115 & 98 & 100 & 64 & 36 & 704 & 110 & 75 & 28 & 13 & 16 & 31 \\
\hline AMG880 & 16,90 & 6,54 & 0,679 & 2,80 & 55,00 & 15,10 & 1,91 & 0,1387 & 0,53 & 0,21 & 146 & 478 & 146 & 99 & 97 & 63 & 30 & 599 & 89 & 66 & 26 & 11 & 15 & 30 \\
\hline AMG881 & 10,82 & 6,76 & 0,764 & 3,06 & 58,85 & 16,71 & 1,95 & 0,1162 & 0,51 & 0,24 & 156 & 417 & 149 & 117 & 109 & 71 & 37 & 866 & 110 & 76 & 28 & 13 & 26 & 34 \\
\hline AMG886 & 18,12 & 5,85 & 0,696 & 2,68 & 55,52 & 13,97 & 2,09 & 0,1615 & 0,37 & 0,35 & 159 & 611 & 125 & 111 & 132 & 56 & 40 & 435 & 84 & 62 & 25 & 9 & 86 & 55 \\
\hline
\end{tabular}

\section{Bibliographie}

BAudoux J., 1996. Les amphores du nord-est de la Gaule (territoire français). Contribution à l'histoire de l'économie provinciale sous l'empire romain. DAF, 52, Paris, Maison des sciences de l'homme.

Benoît F., 1938. Compte rendu des recherches faites à Trinquetaille en 1938. Comptes rendus des séances de l'Académie des Inscriptions et Belles-Lettres, 82, p. 541-548.

Bertucchi G., 1992. Les amphores et le vin de Marseille, vI s. av. J.-C.-II s. apr. J.-C. RAN, suppl. 25.

Bigot F., 2015. Le four FR12005 de l'atelier de potiers d'Espeyran à Saint-Gilles-du-Gard (Gard). Rapport de sondage, Lattes.

Bigot F., à paraître. Nouvelles données, nouvelles réflexions sur la production et la diffusion des amphores gauloises à partir des contextes portuaires et littoraux de Gaule Narbonnaise (Ir s. av.-IV s. apr. J.-C.). Thèse de doctorat, université Montpellier Paul-Valéry.

Bigot F., Djaour D., 2013. Étude préliminaire des amphores gauloises des fouilles de l'épave Arles-Rhône 3 (Arles, Bouches-duRhône) ( $2^{\mathrm{e}}$ moitié du I ${ }^{\mathrm{er}}$ s. $-1^{\mathrm{re}}$ moitié du II ${ }^{\mathrm{e}}$ s. apr. J.-C.). Revue archéologique de Narbonnaise, 46, p. 375-394.

Brentchaloff D., 1988. L'amphore à saumure de type FréjusLenzbourg. SFECAG, Actes du congrès d'Orange, Marseille, p. 179-186.

Brun J.-P., Laubenheimer F., 2001. Conclusions. Gallia, 58, p. $5-260$.

Buffat L., 2011. L'économie domaniale en Gaule Narbonnaise. Monographie d'archéologie méditerranéenne, 29, Lattes.

Buxeda i Garrigós J., Mommsen H., Tsolakidou A., 2002. Alternation of $\mathrm{Na}, \mathrm{K}$, and $\mathrm{Rb}$ concentrations in Mycenaean pottery and a proposed explanation using X-ray diffraction. Archeometry, 44 (2), p. 187-198.

Christol M., Compan E., Roure R., Scrinzi M., Vaschalde C., 2011. Nouvelles données sur l'occupation romaine du comptoir protohistorique d'Espeyran (Saint-Gilles-du-Gard) : découverte d'une inscription de la gens Calvia. Revue archéologique de Narbonnaise, 44, p. 155-162.
Christol M., Fiches J.-L., 1999. Le Rhône : batellerie et commerce dans l'Antiquité. Gallia, 56, p. 141-155.

Corbeel S., en cours : Les producteurs de tuiles et d'amphores de Gaule Narbonnaise. L'apport des estampilles à la connaissance des structures économiques d'une province romaine ( $I^{\text {r }}$ s. av. J.-C.début IV s. apr. J.-C). Thèse de doctorat, université Montpellier Paul-Valéry.

Corbeel S., Duperron G., avec la coll. de Bigot F., Long L., 2013. Nouvelles données sur les timbres sur amphores et couvercles gaulois d'Arles. Revue archéologique de Narbonnaise, 46, p. 395-430.

Duperron G., 2014. Arles et Lyon, ports fluviaux de l'Empire romain Le commerce sur l'axe rhodanien du $I^{e r}$ s. av. J.-C. au VII s. apr. J.-C. Thèse de Doctorat, Université Montpellier Paul-Valéry.

Excoffon P., 2009. Les ateliers de potiers de Provence. Inventaire raisonné. In M. Pasqualini (dir.), Les céramiques communes d'Italie et de Narbonnaise : structures de production, typologies et contextes inédits : II s. av. J.-C.-III s. apr. J.-C., Naples, p. 77-93.

FitzPATrick A., 1992. La place des amphores dans l'approvisionnement militaire de l'Écosse romaine. In F. Laubenheimer (dir.), Les amphores en Gaule. Production et circulation, Table ronde internationale, Metz 4-6 octobre 1990, Paris, p. 179-183.

Gateau F., Gazenbeex M., 1999. Carte Archéologique de la Gaule, 13/2, Les Alpilles et la Montagnette. Paris, Académie des Inscriptions et Belles-Lettres.

Grenier A., 1934. Manuel d'archéologie gallo-romaine. 2. L'archéologie du sol. Paris.

Ignace J.-M., 2001. Les Tourelles, Beaucaire, Évaluation archéologique 2001. Document Final de Synthèse dactyl., inédit, Beaucaire.

Ignace J.-M., 2002. Les Tourelles, Beaucaire, Fouille programmée 2002, Document Final de Synthèse dactyl., inédit, Beaucaire.

Ignace J.-M., 2003. Les Tourelles, Beaucaire, Fouille programmée 2003, Document Final de Synthèse dactyl., inédit, Beaucaire.

Ignace J.-M., 2004. Les Tourelles, Beaucaire, Fouille programmée 2004, Document Final de Synthèse dactyl., inédit, Beaucaire. 
Laubenheimer F., 1985. La production des amphores en Gaule Narbonnaise sous le Haut Empire. Les Belles Lettres, Paris.

Laubenheimer F., 1989. Les amphores gauloises sous l'Empire : recherches nouvelles sur leur production et leur chronologie. In Amphores romaines et Histoire économique : dix ans de recherches, Actes du colloque de Sienne (22-24 mai 1986). Rome, Collection de l'École française de Rome, p. 105-138.

Laubenheimer F., 2001. Le vin gaulois de Narbonnaise exporté dans le monde romain sous le Haut-Empire. In F. Laubenheimer (dir.), 20 ans de recherches à Sallèles d'Aude, PUFC, Besançon, p. 51-65.

Laubenheimer F., Gebara C., Beraud I., 1992. Production d'amphores à Fréjus. In F. Laubenheimer (dir.), Les amphores en Gaule, production et circulation, Table ronde internationale, Metz 4-6 octobre 1990, Paris, 1992, p. 15-24.

Laubenheimer F., Schmitt A., 2009. Amphores vinaires de Narbonnaise, Production et grand commerce. Création d'une base de données géochimiques des ateliers. Lyon, TMO.

Lautier L., Rothé M.-P., 2010. Carte Archéologique de la Gaule, 06, Les Alpes-Maritimes. Paris, Académie des Inscriptions et Belles Lettres.

Long L., 1992. Arles. Lit du Rhône. Gallia Informations. Préhistoire et Histoire, p. 9-14.

Long L., Duperron G., 2011. Le mobilier de la fouille de l'épave romaine Arles-Rhône 7. Un navire fluvio-maritime du III siècle de notre ère. SFECAG, Actes du Congrès d'Arles, Marseille, p. 37-56.

Long L., Duperron G. avec la coll. de Bonifay M., Capelli C., Desbat A., et Leger C., 2013 . Navigation et commerce dans le delta du Rhône : l'épave Arles-Rhône 14 (III s. apr. J.-C.). In S. Mauné, G. Duperron (éd.), Du Rhône aux Pyrénées. Aspects de la Vie Matérielle en Gaule Narbonnaise II, éd. M. Mergoil (coll. Archéologie et Histoire Romaine, 25), Montagnac, p. $125-167$.

Long L., Duperron G., 2014. Note préliminaire sur l'épave Arles-Rhône 13. Un navire de mer en contexte fluvial, à Arles, au $\mathrm{IV}^{\mathrm{e}}$ siècle apr. J.-C. Cahiers d'Archéologie Subaquatique, 22, p. 115-144.

Marty F. 2003. L'atelier de potiers gallo-romain de Sivier (Istres, Bouches-du-Rhône). Revue archéologique de Narbonnaise, 36, p. 256-282.
Mauné S., 2013. La géographie des productions des ateliers d'amphores de Gaule Narbonnaise pendant le Haut-Empire. Nouvelles données et perspectives. Revue archéologique de Narbonnaise, 46, p. 335-374.

Mauné S., Garcia Vargas E., Bourgeon O., Corbeel S., Carrato C., Garcia Dils S., Bigot F., Vazquez Paz J., 2014. L'atelier d'amphores à huile Dr. 20 de Las Delicias à Ecija (prov. de Séville, Espagne). SFECAG, Actes du congrès de Chartres, Marseille, p. 419-444.

Mauné S., Silvéréano S., avec la coll. de Newman Ch., 2011. Les productions augusto-tibériennes de l'atelier de potiers de Bastide-Neuve à Velaux (B.-du-Rh.). SFECAG, Actes du congrès d'Arles, Marseille, p. 362-389.

Mouraret J., 2002-2003. Une série de timbres sur amphore à Caumont-sur-Durance (Vaucluse). Bulletin archéologique de Provence, 31-32, p. 101-107.

PANella Cl., 1992. Mercato di Roma e anfora galliche nella prima età imperiale. In F. Laubenheimer (dir.), Table ronde internationale, Metz 4-6 octobre 1990, Les amphores en Gaule. Production et circulation, Paris, p. 185-205.

Panella Cl., Rizzo G., 2014. Ostia VI. Les terme del Nuotare. Roma, L'Erma di Bretschneider.

Picon M., 1976. Remarques préliminaires sur deux types d'altération de la composition chimique des céramiques au cours du temps. Figlina, 1, 1976, p.159-166.

Provost M., et al., 1999. Le Gard, 30/2-3, Carte Archéologique de la Gaule. Paris, Académie des Inscriptions et Belles Lettres.

Rivet L., 1986. Un atelier de potiers du $\mathrm{I}^{\text {er }}$ siècle de notre ère à Mandelieu (A.-M.). DAF, 9, p. 119-134.

Rothé M.-P., Heijmans M., 2008. Carte Archéologique de la Gaule, 13/5, Arles, Crau, Camargue. Paris, Académie des Inscriptions et Belles Lettres.

Schwedt A., Mommsen H., Zacharias N., Buxeda i Garrigós J., 2006. Anacilme crystallization and compositional profilescomparing approaches to detect post-depositional alternations in archeological pottery. Archeometry, 48 (2), p. 237-251.

Tchernia A., 2011. Les Romains et le commerce. Études du Centre Jean-Bérard 8, Naples, 439 p. 